\title{
Non-uniform non-tensor product local interpolatory subdivision surfaces
}

\author{
Carolina Vittoria Beccari, ${ }^{*}$, Giulio Casciola ${ }^{\mathrm{a}}$, Lucia Romani ${ }^{\mathrm{b}}$ \\ ${ }^{a}$ Department of Mathematics, University of Bologna, P.zza Porta San Donato 5, 40127 Bologna, Italy \\ ${ }^{b}$ Department of Mathematics and Applications, University of Milano-Bicocca, Via R. Cozzi 53, 20125 Milano, Italy
}

\begin{abstract}
In this paper we exploit a class of univariate, $C^{1}$ interpolating four-point subdivision schemes featured by a piecewise uniform parameterization, to define non-tensor product subdivision schemes interpolating regular grids of control points and generating $C^{1}$ limit surfaces with a better behavior than the well-established tensor product subdivision and spline surfaces. As a result, it is emphasized that subdivision methods can be more effective than splines, not only, as widely acknowledged, for the representation of surfaces of arbitrary topology, but also for the generation of smooth interpolants of regular grids of points.

To our aim, the piecewise uniform parameterization of the univariate case is generalized to an augmented parameterization, where the knot intervals of the $k$ th level grid of points are computed from the initial ones by an updating relation that keeps the subdivision algorithm linear. The particular parameters configuration, together with the structure of the subdivision rules, turn out to be crucial to prove the continuity and smoothness of the limit surface.
\end{abstract}

Key words: Interpolation, Subdivision, Non-tensor product surfaces, Non-uniform parameterization.

\section{Introduction}

Subdivision curves and surfaces have so far become an effective alternative to the well known parametric splines. While approximating subdivision schemes represent a generalization of either uniform or non-uniform splines [10, $22,25,26]$, this is no longer true for interpolating subdivision methods, which require ad hoc definition and analysis [1-5, 11-14, 17, 21, 23, 24]. In this respect, uniform interpolatory schemes have been investigated in detail, both for the curve and surface cases, and by now fully understood. Interpolatory methods with non-uniform parameterization, were introduced in the seminal work by Daubechies et al. [16] and more recently they have been the topic of several papers (see $[6,7,17])$. This renewed interest has arisen since it was observed that the non-uniform parameterization may significantly reduce interpolation artifacts (like unwanted undulation, cusps and self-intersections) with respect to the uniform. This behavior is analogous to that of splines, illustrated with an example in Figure 1, where the class of locally supported cardinal spline functions in [15] is exploited. According to several works, the centripetal parameterization appears to be the best for curve interpolation. In [8] the authors show that for a whole family of non-uniform interpolating splines with different approximation order, support width and continuity the centripetal parameterization provides a good-looking interpolant, avoiding or minimizing interpolation artifacts. Moreover [20] gives a formal mathematical explanation of the fact that, for cubic splines, such parameterization significantly bounds the global and local deviation of the resulting curve from its data polygon. In a similar way, it is proven in [28] that, for cubic Catmull-Rom curves, the centripetal parameterization is the only one to guarantee no cusps and selfintersections within curve segments. As concerns interpolating subdivision curves, a result confirming the advantage of the centripetal parameterization was presented in [17] where, the authors propose a non-linear 4-point scheme derived by up-sampling from the cubic non-uniform Lagrange interpolant, with underlying parameterization recomputed at each subdivision step. For such scheme, the uniform, chordal and centripetal parameterizations have been compared, showing that the centripetal one minimizes the distance between the data polygon and the limit curve.

\footnotetext{
${ }^{*}$ Corresponding author.

Email addresses: carolina.beccari2@unibo.it (Carolina Vittoria Beccari), giulio.casciola@unibo.it (Giulio Casciola), lucia.romani@unimib.it (Lucia Romani)

Preprint submitted to Elsevier

October 12, 2012
} 

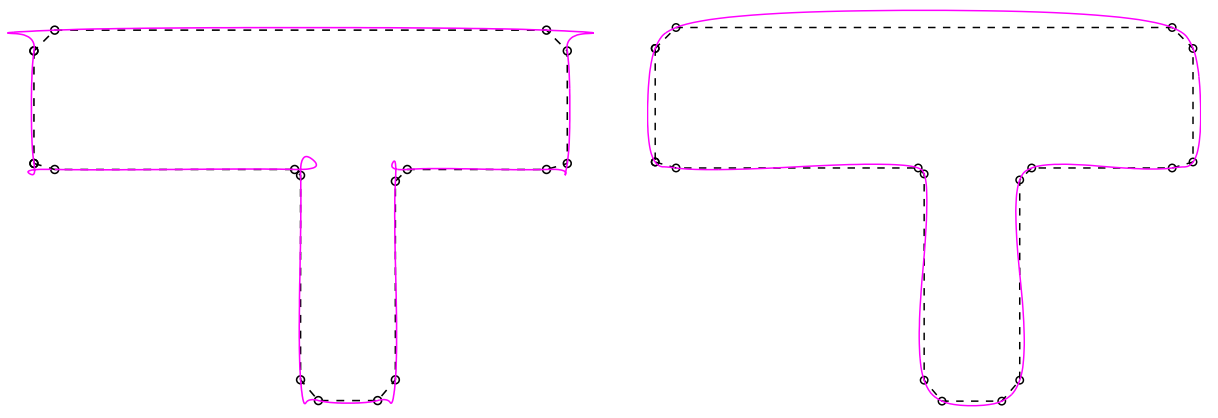

Figure 1: Comparison between quadratic spline curves interpolating highly non-uniform data through uniform (left) and centripetal (right) parameterization.

Our work originates from the observation that, even if based on the centripetal parameterization, a tensor product spline surface may not generate a good quality interpolant. To understand why, let us denote by $\left\{p_{i, j}\right\}_{i, j \in \mathbb{Z}}$ the vertices of the given regular quadrilateral mesh and by $\left\{x_{i, j}\right\}$ the associated non-uniform parameter values (also called knots). In order to compute the tensor product surface interpolating the vertices $p_{i, j}$ in correspondence to the parameters $x_{i, j}$, it is necessary to work out only two parameter sets corresponding to the two grid directions. The solving strategy is to compute the average of the parameter values $x_{i, j}$, previously defined along the two directions, thus loosing information that turn out to be crucial to the quality of the interpolant. In particular, each section curve of the mesh is not allowed to maintain its own non-uniform parameterization, as would be especially desirable in the interpolation context, but it is parameterized by the average of the parameterizations of all section curves in the related grid direction. The fact that the strict structure of the tensor product may compromise the quality of the interpolation is well-known, see e.g. [19], §7.5.1.

The main novelty of this paper is a method to get a good quality surface interpolating the vertices of a regular quadrilateral mesh: the proposed approach is based on a non-uniform, non-tensor product interpolatory subdivision algorithm where, instead of constraining the parameterization to the tensor product structure, a possibly different knot interval can be associated to each edge of the initial control mesh. In this way, each section polyline is individually interpolated together with its parameters, thus maintaining its own parameterization.

For approximation purposes, a similar non-tensor product configuration was firstly studied in [26] and most recently in $[10,22]$, where the authors propose non-uniform subdivision algorithms based on biquadratic and bicubic B-splines. In the interpolatory context, this paper is the first addressing a non-tensor product setting. The non-uniform, nontensor product surface subdivision scheme is defined as a generalization of a class of piecewise uniform, univariate, interpolatory 4-point schemes derived by upsampling fundamental bases for interpolation, as those in [6, 8, 16]. As a consequence, the coefficients of the scheme depend on the non-uniform, local knot intervals upon which the underlying cardinal spline basis is defined. These univariate schemes, termed reference schemes, are exploited as a basis to derive refinement rules for regular grids, that, besides exploiting the advantages of the centripetal parameterization, are linear and capable of generating $C^{1}$ continuous surfaces of good quality. To this aim, the piecewise uniform parameterization of the univariate reference scheme is generalized to a so called augmented parameterization, where the parameters of the $k$ th level grid of points are computed from the initial ones by an updating relation that keeps the subdivision algorithm linear. To prove continuity and smoothness of the limit surface we strongly rely on the fact that the scheme interpolates all the section polylines that are created at each step and we exploit the linearity of the subdivision rules as well as the asymptotical behavior of the parameterization.

The remainder of the paper is organized as follows. In Section 2 we recall the refinement rules of the class of non-uniform $C^{1}$ interpolatory 4-point schemes that can be conveniently exploited as reference schemes. In Section 3 we describe the key ideas at the basis of our non-uniform, non-tensor product interpolatory subdivision scheme for regular quadrilateral meshes and we explain in detail the edge and face point rules it relies upon. Then, in Section 4 we perform the continuity and smoothness analysis of the proposed surface scheme and in Section 5 we show some numerical examples confirming the effectiveness of our proposal and its advantages with respect to non-uniform tensor product subdivisions and splines. A summary of the main contributions of this paper and the outline of our future 
work are described in Section 6.

\section{A class of non-uniform interpolatory 4-point schemes}

The novel surface scheme we aim to define is conceived as a generalization of a univariate, non-uniform interpolatory 4-point scheme, that we call the reference scheme. Thus, before defining the surface refinement rules, we briefly overview the related univariate method.

Denoted by $\left\{p_{i}^{0}\right\}$ the vertices of the initial polyline and by $\left\{x_{i}^{0}\right\}$ the associated parameter values, also called knots, for all $k \geqslant 0$ the general refinement equations of a non-uniform interpolatory 4-point scheme are

$$
\begin{aligned}
& p_{2 i}^{k+1}=p_{i}^{k}, \\
& p_{2 i+1}^{k+1}=a_{0, i}^{k} p_{i-1}^{k}+a_{1, i}^{k} p_{i}^{k}+a_{2, i}^{k} p_{i+1}^{k}+a_{3, i}^{k} p_{i+2}^{k},
\end{aligned}
$$

where the coefficients $a_{\ell, i}^{k}, \ell=0, \ldots, 3$, depend on the knots $\left\{x_{i}^{k}\right\}$ of the $k$ th level polyline. In order to emphasize the locality of the scheme, it is convenient to rewrite these coefficients in terms of the length of the three consecutive knot intervals $d_{j}^{k}:=x_{j+1}^{k}-x_{j}^{k}, j=i-1, i, i+1$, introducing the notation

$$
a_{\ell}\left(\left[d_{i-1}^{k}, d_{i}^{k}, d_{i+1}^{k}\right]\right), \quad \ell=0, \ldots, 3 .
$$

In this paper we focus on reference schemes whose coefficients can be obtained by fitting a local analytic interpolant through the points $\left(x_{i+\ell-1}^{k}, p_{i+\ell-1}^{k}\right), \ell=0, \ldots, 3$, and evaluating it at an arbitrary parameter value inside the central interval $\left[x_{i}^{k}, x_{i+1}^{k}\right]$. From this point onward we restrict our attention to the category of the so called semi-regular insertion rules, where the coefficients $a_{\ell, i}^{k}, \ell=0, \ldots, 3$, are obtained by evaluating the considered interpolant at the parameter value $\left(x_{i}^{k}+x_{i+1}^{k}\right) / 2$. For these schemes, denoted by $\left\{d_{i}^{0}\right\}$ the set of initial knot intervals, we compute the knot intervals for the successive levels through the formula

$$
d_{2 i}^{k+1}=d_{2 i+1}^{k+1}=\frac{d_{i}^{k}}{2}, \quad k \geqslant 0
$$

which simulates a recursive midpoint refinement of the associated parameterization. Thanks to this updating rule, the subdivision algorithm is linear and, after a few rounds of subdivision, the knot intervals assume a piecewise uniform configuration, namely the parameterization is uniform everywhere except at isolated points corresponding to the initial polyline vertices. Schemes of this kind have been the topic of several papers including [6, 7, 16, 27]. In this case the insertion rule in (1) can be written as

$$
p_{2 i+1}^{k+1}=\sum_{\ell=0}^{3} \psi_{i+\ell-1}\left(\frac{x_{i}^{k}+x_{i+1}^{k}}{2}\right) p_{i+\ell-1}^{k}
$$

where $\psi_{i+\ell-1}, \ell=0, \ldots, 3$, are non-uniform cardinal basis functions defined on $\left\{x_{i}^{k}\right\}$ which

(i) satisfy the cardinality condition

$$
\psi_{i+\ell-1}\left(x_{j}^{k}\right)=\left\{\begin{array}{ll}
1 & \text { if } i+\ell-1=j, \\
0 & \text { otherwise },
\end{array} \quad \text { for } j=i-1, \cdots, i+2 ;\right.
$$

(ii) are globally $C^{1}$;

(iii) are either polynomials or piecewise polynomials in $\Pi_{\rho}$ such that for any $f \in \Pi_{\rho}$,

$$
f(x)=\sum_{\ell=0}^{3} \psi_{i+\ell-1}(x) f\left(x_{i+\ell-1}^{k}\right), \quad x \in\left[x_{i}^{k}, x_{i+1}^{k}\right] .
$$


Examples of such fundamental functions can be found in $[6,8]$ amongst others.

Since on the interval $\left[x_{i}^{k}, x_{i+1}^{k}\right]$ the basis $\psi_{i+\ell-1}, \ell=0, \cdots, 3$, is invariant under uniform scaling of knots, the resulting interpolant depends only on the local configuration of the knot intervals. Thus indicated the local parameter triple by

$$
\delta_{i}^{k}:=\left[d_{i-1}^{k}, d_{i}^{k}, d_{i+1}^{k}\right],
$$

we introduce the notation

$$
\psi_{\ell}^{\delta_{i}^{k}}:=\psi_{i+\ell-1}, \quad \ell=0, \ldots, 3 .
$$

Moreover, without loss of generality, we can assume $x_{i-1}^{k}=0$ such that the coefficients $a_{\ell, i}^{k}, \ell=0, \cdots, 3$, are equivalently given by

$$
a_{\ell}\left(\boldsymbol{\delta}_{i}^{k}\right)=\psi_{\ell}^{\boldsymbol{\delta}_{i}^{k}}\left(m_{\boldsymbol{\delta}_{i}^{k}}\right) \quad \text { with } m_{\boldsymbol{\delta}_{i}^{k}}=d_{i-1}^{k}+\frac{1}{2} d_{i}^{k} .
$$

We observe that any analytic interpolant obtained from basis functions satisfying properties (i)-(iii) gives rise to a different set of coefficients, so that a large number of reference schemes fitting into the considered framework is available. However, to design a $C^{1}$ surface subdivision scheme it is also necessary that the reference scheme be $C^{1}$. We conclude this section by providing two examples of schemes that satisfy all the stated requirements. For instance, if we exploit non-uniform cubic Lagrange interpolation, as in [16], the derived coefficients are

$$
\begin{aligned}
& a_{0}\left(\boldsymbol{\delta}_{i}^{k}\right)=-\frac{\left(d_{i}^{k}\right)^{2}\left(d_{i}^{k}+2 d_{i+1}^{k}\right)}{8 d_{i-1}^{k}\left(d_{i-1}^{k}+d_{i}^{k}\right)\left(d_{i-1}^{k}+d_{i}^{k}+d_{i+1}^{k}\right)}, \\
& a_{1}\left(\boldsymbol{\delta}_{i}^{k}\right)=\frac{\left(d_{i}^{k}\right)^{2}+2\left(d_{i-1}^{k}+d_{i+1}^{k}\right) d_{i}^{k}+4 d_{i-1}^{k} d_{i+1}^{k}}{8 d_{i-1}^{k}\left(d_{i}^{k}+d_{i+1}^{k}\right)}, \\
& a_{2}\left(\boldsymbol{\delta}_{i}^{k}\right)=\frac{\left(d_{i}^{k}\right)^{2}+2\left(d_{i-1}^{k}+d_{i+1}^{k}\right) d_{i}^{k}+4 d_{i-1}^{k} d_{i+1}^{k}}{8 d_{i+1}^{k}\left(d_{i-1}^{k}+d_{i}^{k}\right)}, \\
& a_{3}\left(\boldsymbol{\delta}_{i}^{k}\right)=-\frac{\left(d_{i}^{k}\right)^{2}\left(2 d_{i-1}^{k}+d_{i}^{k}\right)}{8 d_{i+1}^{k}\left(d_{i}^{k}+d_{i+1}^{k}\right)\left(d_{i-1}^{k}+d_{i}^{k}+d_{i+1}^{k}\right)},
\end{aligned}
$$

while if we interpolate through the non-uniform quadratic fundamental splines in [15] we obtain the set of coefficients

$$
\begin{aligned}
& a_{0}\left(\delta_{i}^{k}\right)=-\frac{\left(d_{i}^{k}\right)^{2}}{8 d_{i-1}^{k}\left(d_{i-1}^{k}+d_{i}^{k}\right)}, \\
& a_{1}\left(\delta_{i}^{k}\right)=\frac{\left(d_{i}^{k}\right)^{2}+d_{i}^{k}\left(3 d_{i-1}^{k}+d_{i+1}^{k}\right)+4 d_{i-1}^{k} d_{i+1}^{k}}{8 d_{i-1}^{k}\left(d_{i}^{k}+d_{i+1}^{k}\right)}, \\
& a_{2}\left(\delta_{i}^{k}\right)=\frac{\left(d_{i}^{k}\right)^{2}+d_{i}^{k}\left(d_{i-1}^{k}+3 d_{i+1}^{k}\right)+4 d_{i-1}^{k} d_{i+1}^{k}}{8 d_{i+1}^{k}\left(d_{i}^{k}+d_{i-1}^{k}\right)}, \\
& a_{3}\left(\delta_{i}^{k}\right)=-\frac{\left(d_{i}^{k}\right)^{2}}{8 d_{i+1}^{k}\left(d_{i}^{k}+d_{i+1}^{k}\right)},
\end{aligned}
$$

which was proposed in [6]. By construction, the two reference schemes with coefficients in (5) and (6) reproduce polynomials respectively in $\Pi_{3}$ and $\Pi_{2}$; moreover, in the referenced papers, it was proven that they are $C^{1}$ when the parameterization is piecewise uniform. Being these schemes stationary and uniform everywhere but at isolated points, 
corresponding to the initial polyline vertices, their continuity and smoothness can be proved by spectral analysis of the local subdivision matrix, as proposed in [27].

\section{The Non-Uniform Local Interpolatory Subdivision Scheme (NULISS) for regular quadrilateral meshes}

The existing literature has always considered subdivision schemes as advantageous alternatives to tensor product constructions due to their ability to deal with extraordinary points and model surfaces of arbitrary topology. Conversely, in this paper, we want to show that subdivision may also perform significantly better than tensor product splines also in the context of interpolation of regular quadrilateral meshes. The goal of this section is to provide a general formulation of a 4-point based interpolatory subdivision scheme for regular quadrilateral meshes that offers a very efficient tool for interpolating a given grid of points taking into account the associated parameter values. Hereinafter this scheme is called NULISS (Non-Uniform Local Interpolatory Subdivision Scheme). Its insertion rules are conceived as a natural extension to the regular quadrilateral mesh of a non-uniform interpolatory 4-point reference scheme with the requirements stated in Section 2.

Let $\mathcal{M}^{0}$ denote a regular quadrilateral grid of 3D points. Denoted by $\left\{p_{i, j}^{0}\right\}_{i, j \in \mathbb{Z}}$ the vertices of the mesh, we associate with each edge along one grid direction a knot interval $d_{i, j}^{0}=\left\|p_{i, j+1}^{0}-p_{i, j}^{0}\right\|_{2}^{\frac{1}{2}}$, according to the centripetal parameterization. Analogously, along the other direction, we define knot intervals $e_{i, j}^{0}=\left\|p_{i+1, j}^{0}-p_{i, j}^{0}\right\|_{2}^{\frac{1}{2}}$. In the following we consider an iterative subdivision algorithm that takes as input the mesh $\mathcal{M}^{0}$ with the associated initial knot intervals and generates in the limit a smooth surface based on the steps outlined below.

Algorithm 1. For each refinement level $k \geqslant 1$, it

1. retains each vertex point (Figure 2-left, green bullets);

2. computes a new edge point for each edge, using the univariate reference scheme (Figure 2-left, magenta bullets);

3. computes a new face point for each face (Figure 2-left, blue bullets);

4. creates new edges by connecting each new face point to the new edge points of the edges surrounding the face, and connecting each vertex point to the new edge points of the edges incident on that vertex;

5. creates new faces that have a loop of four new edges;

6. computes the knot interval values for the refined mesh and assigns them to the new edges.

The above steps 1.,2.,3. define the new geometry and steps 4., 5. define the connectivity. When this process step continues, it yields a sequence of refined meshes which converges to a limit surface.

Vertex, edge and face points are determined by the equations

- vertex points $p_{2 i, 2 j}^{k+1}=p_{i, j}^{k}$;

- edge points $p_{2 i+1,2 j}^{k+1}=\sum_{r=0}^{3} b_{r, i, j}^{k} p_{i+r-1, j}^{k}$ and $p_{2 i, 2 j+1}^{k+1}=\sum_{\ell=0}^{3} c_{\ell, i, j}^{k} p_{i, j+\ell-1}^{k}$;

- face points $p_{2 i+1,2 j+1}^{k+1}=\sum_{r=0}^{3} \sum_{\ell=0}^{3} b_{r, i, j}^{k} c_{\ell, i, j}^{k} p_{i+r-1, j+\ell-1}^{k}$.

As previously mentioned, in the tensor product case we need to define an average parameterization where

$$
\bar{d}_{j}^{k}=\frac{\sum_{i \in \mathbb{Z}} d_{i, j}^{k}}{\sharp\left\{d_{i, j}^{k} \mid i \in \mathbb{Z}\right\}} \quad \text { and } \quad \bar{e}_{i}^{k}=\frac{\sum_{j \in \mathbb{Z}} e_{i, j}^{k}}{\sharp\left\{e_{i, j}^{k} \mid j \in \mathbb{Z}\right\}},
$$



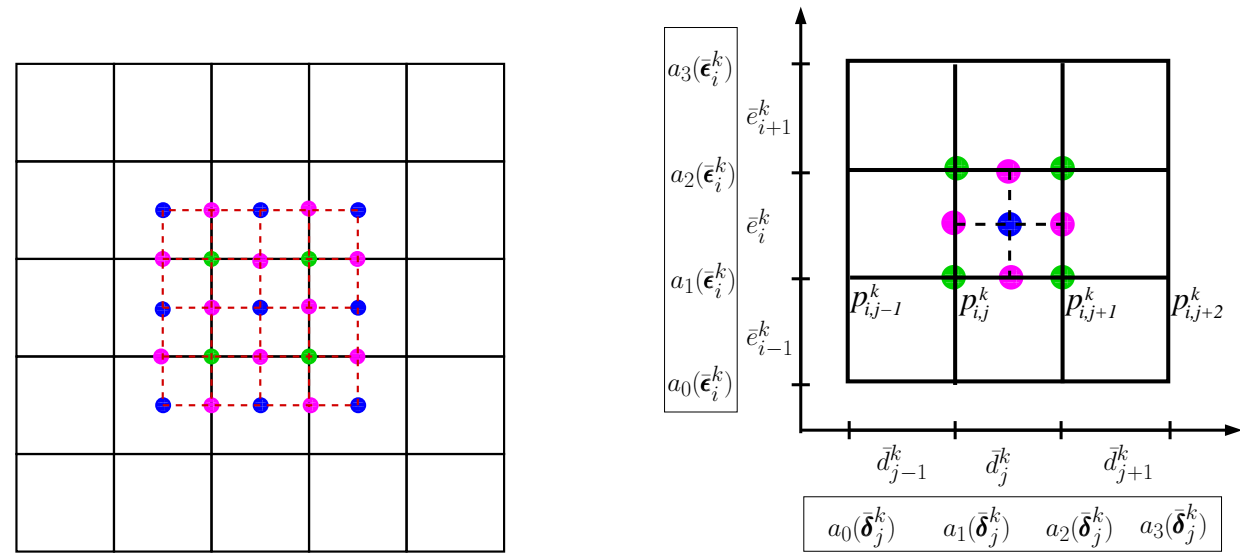

Figure 2: Left: One step of the considered interpolatory subdivision algorithm, the initial mesh is in black, while the refined mesh in red. Green, magenta and blue bullets represent vertex, edge and face points, respectively. Right: Parameters configuration and related coefficients for the tensor product scheme.

so that $\left\{\bar{d}_{j}^{k}\right\}_{j \in \mathbb{Z}}$ and $\left\{\bar{e}_{i}^{k}\right\}_{i \in \mathbb{Z}}$ are the knot intervals associated with the two grid directions (see Figure 2-right for a graphical interpretation). Thus, according to (4), the coefficients $b_{r, i, j}^{k}$ and $c_{\ell, i, j}^{k}$ are determined by the chosen univariate reference scheme as

$$
\begin{aligned}
& \forall j, \quad b_{r, i, j}^{k}=a_{r}\left(\overline{\boldsymbol{\epsilon}}_{i}^{k}\right)=\psi_{r}^{\overline{\boldsymbol{\epsilon}}_{i}^{k}}\left(m_{\overline{\boldsymbol{\epsilon}}_{i}^{k}}\right), \quad r=0, \cdots, 3, \quad \text { with } \overline{\boldsymbol{\epsilon}}_{i}^{k}=\left[\bar{e}_{i-1}^{k}, \bar{e}_{i}^{k}, \bar{e}_{i+1}^{k}\right] \text { and } m_{\overline{\boldsymbol{\epsilon}}_{i}^{k}}=\bar{e}_{i-1}^{k}+\frac{1}{2} \bar{e}_{i}^{k}, \\
& \forall i, \quad c_{\ell, i, j}^{k}=a_{\ell}\left(\overline{\boldsymbol{\delta}}_{j}^{k}\right)=\psi_{\ell} \overline{\boldsymbol{\delta}}_{j}^{k}\left(m_{\overline{\boldsymbol{\delta}}_{j}^{k}}\right), \quad \ell=0, \cdots, 3, \quad \text { with } \overline{\boldsymbol{\delta}}_{j}^{k}=\left[\bar{d}_{j-1}^{k}, \bar{d}_{j}^{k}, \bar{d}_{j+1}^{k}\right] \text { and } m_{\overline{\boldsymbol{\delta}}_{j}^{k}}=\bar{d}_{j-1}^{k}+\frac{1}{2} \bar{d}_{j}^{k} .
\end{aligned}
$$

The requirement for averaging the parameterization may determine a significant loss of quality in the limit surface. Our goal is thus to generalize the above edge and face point formulas so as to consider a whole set of parameters surrounding the location of insertion.

In order to describe the refinement rules of the sought scheme, we need to properly define steps 2 . and 3 . of the refinement Algorithm 1. Figure 3 illustrates the two steps for one of the faces of $\mathcal{M}^{k}$. A new edge point $E^{k+1}$ is placed in the middle of the edge with vertices $p_{i, j}^{k}$ and $p_{i, j+1}^{k}$, by the formula

$$
E^{k+1}=p_{2 i, 2 j+1}^{k+1}=a_{0}\left(\boldsymbol{\delta}_{i, j}^{k}\right) p_{i, j-1}^{k}+a_{1}\left(\boldsymbol{\delta}_{i, j}^{k}\right) p_{i, j}^{k}+a_{2}\left(\boldsymbol{\delta}_{i, j}^{k}\right) p_{i, j+1}^{k}+a_{3}\left(\boldsymbol{\delta}_{i, j}^{k}\right) p_{i, j+2}^{k},
$$

where coefficients $a_{\ell}\left(\boldsymbol{\delta}_{i, j}^{k}\right), \ell=0, \ldots, 3$, are computed by (4) with $\boldsymbol{\delta}_{i, j}^{k}=\left[d_{i, j-1}^{k}, d_{i, j}^{k}, d_{i, j+1}^{k}\right]$; this means that the new edge point is generated by applying the insertion rule of the univariate reference scheme to the related edge of $\mathcal{M}^{k}$ (see Figure 3-left). The edge points along the other edges can be determined following the same approach.

To explain the insertion of a face point we observe that the vertices of the face of insertion can be seen as the intersection of 4 section polylines, two in each direction (see Fig. 3-right). We denote by

$$
\boldsymbol{\Delta}_{i, j}^{k}=\left[\frac{d_{i, j-1}^{k}+d_{i+1, j-1}^{k}}{2}, \frac{d_{i, j}^{k}+d_{i+1, j}^{k}}{2}, \frac{d_{i, j+1}^{k}+d_{i+1, j+1}^{k}}{2}\right] \quad \text { and } \quad \boldsymbol{E}_{i, j}^{k}=\left[\frac{e_{i-1, j}^{k}+e_{i-1, j+1}^{k}}{2}, \frac{e_{i, j}^{k}+e_{i, j+1}^{k}}{2}, \frac{e_{i+1, j}^{k}+e_{i+1, j+1}^{k}}{2}\right]
$$

the triples of knot intervals that refer to virtual edges (i.e. edges that do not belong to the $k$ th level mesh $\mathcal{M}^{k}$ ) in the two grid directions. They are computed by averaging existing knot intervals on opposite edges of the considered face and of its four adjacent faces, and can be respectively seen as a local parameterization for the two virtual section polylines represented by dashed lines in Fig. 3-right. Recalling equation (4) we can now compute $a_{\ell}\left(\boldsymbol{\Delta}_{i, j}^{k}\right)$ and $a_{\ell}\left(\boldsymbol{E}_{i, j}^{k}\right)$, $\ell=0, \ldots, 3$, and determine the location of each face point $F^{k+1}=p_{2 i+1,2 j+1}^{k+1}$ as 

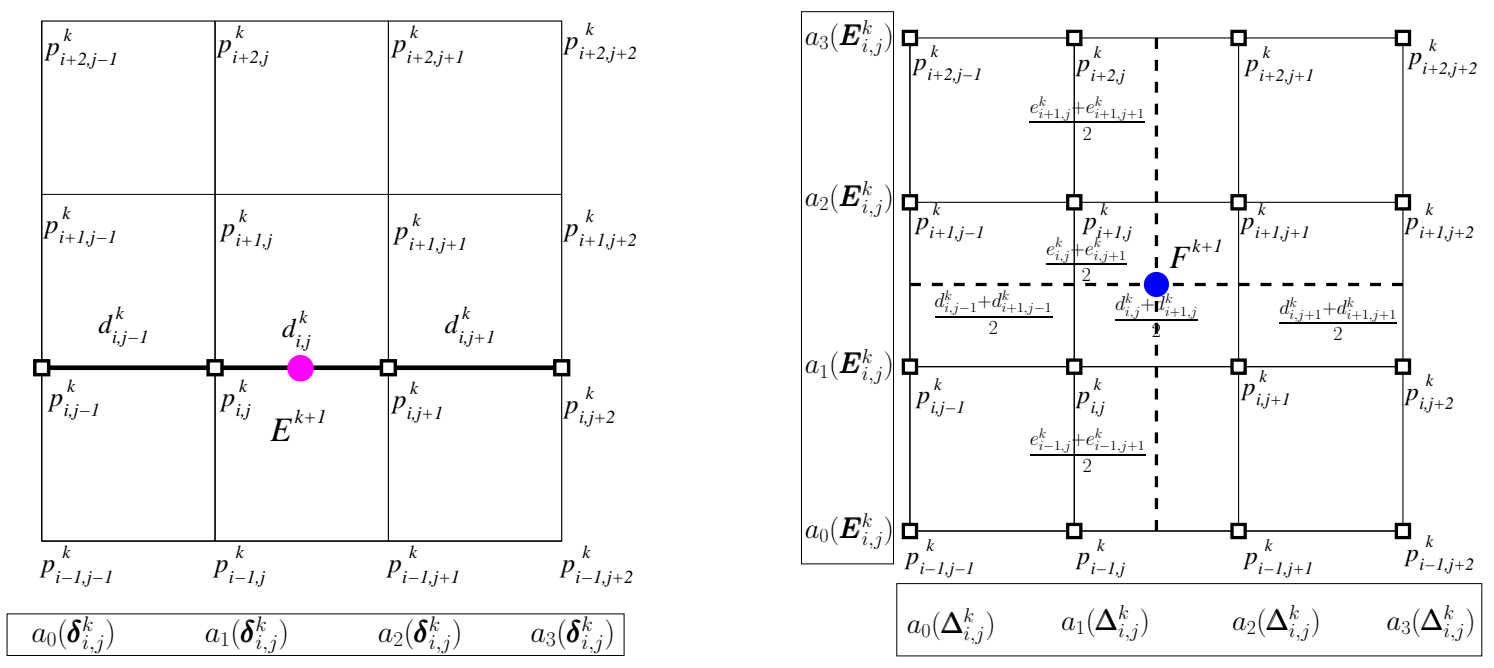

Figure 3: Edge point rule (left) and face point rule (right) for the NULISS scheme.

$$
F^{k+1}=\left[a_{0}\left(\boldsymbol{E}_{i, j}^{k}\right), a_{1}\left(\boldsymbol{E}_{i, j}^{k}\right), a_{2}\left(\boldsymbol{E}_{i, j}^{k}\right), a_{3}\left(\boldsymbol{E}_{i, j}^{k}\right)\right]\left[\begin{array}{cccc}
p_{i-1, j-1}^{k} & p_{i-1, j}^{k} & p_{i-1, j+1}^{k} & p_{i-1, j+2}^{k} \\
p_{i, j-1}^{k} & p_{i, j}^{k} & p_{i, j+1}^{k} & p_{i, j+2}^{k} \\
p_{i+1, j-1}^{k} & p_{i+1, j}^{k} & p_{i+1, j+1}^{k} & p_{i+1, j+2}^{k} \\
p_{i+2, j-1}^{k} & p_{i+2, j}^{k} & p_{i+2, j+1}^{k} & p_{i+2, j+2}^{k}
\end{array}\right]\left[\begin{array}{c}
a_{0}\left(\boldsymbol{\Delta}_{i, j}^{k}\right) \\
a_{1}\left(\boldsymbol{\Delta}_{i, j}^{k}\right) \\
a_{2}\left(\boldsymbol{\Delta}_{i, j}^{k}\right) \\
a_{3}\left(\boldsymbol{\Delta}_{i, j}^{k}\right)
\end{array}\right]
$$

Figure 3-right illustrates the insertion of the considered face point.

We immediately observe that, oppositely to the tensor product construction, the edge point rules of NULISS ensure that the vertices that describe each section polyline of the mesh are interpolated at the corresponding centripetal parameters. In this way, each section curve of the limit surface maintains its own parameterization (instead of being parameterized by the global average of the parameterizations in the related grid direction) and thus it has the optimal behavior guaranteed by the centripetal parameterization.

Moreover the face point insertion rule of NULISS does not involve the complete set of parameters associated with the $4 \times 4$ grid of vertices that determines a new face point, but only the parameters of the vertices related to the face of insertion and to the four adjacent faces. In fact, it makes sense to require that the virtual parameterization adopted to insert a face point, does not significantly deviate from the parameterization of the section curves that define a face. Conversely taking into account the whole $4 \times 4$ grid of parameters may generate undesired distortions in the parameterization.

So far we have described steps 1.-5. in Algorithm 1. The following subsection illustrates the strategy pursued to accomplish step 6. of the considered subdivision algorithm.

\subsection{The augmented parameterization and its parameters updating rule}

Each subdivision step generates a refined mesh, with more vertices, edges and faces, and as a consequence a suitable parameterization should be set in correspondence to the newly created edges. The method that we choose to compute the values of the knot intervals influences the linearity and stationarity of the scheme as well as the properties of the limit shape, thus it should be carefully devised. In order to guarantee that the refinement rules of NULISS identify a linear subdivision process, we use an updating strategy to deduce the $k$ th level knot intervals from those computed at level 0 . The method to update the parameters from level 0 to 1 is shown in Fig. 4 and can be repeated iteratively at each step $k \geqslant 1$ : the knot intervals defined in correspondence to edges of the coarse mesh are halved and duplicated, while those in correspondence to a new edge created inside a face are obtained by averaging 

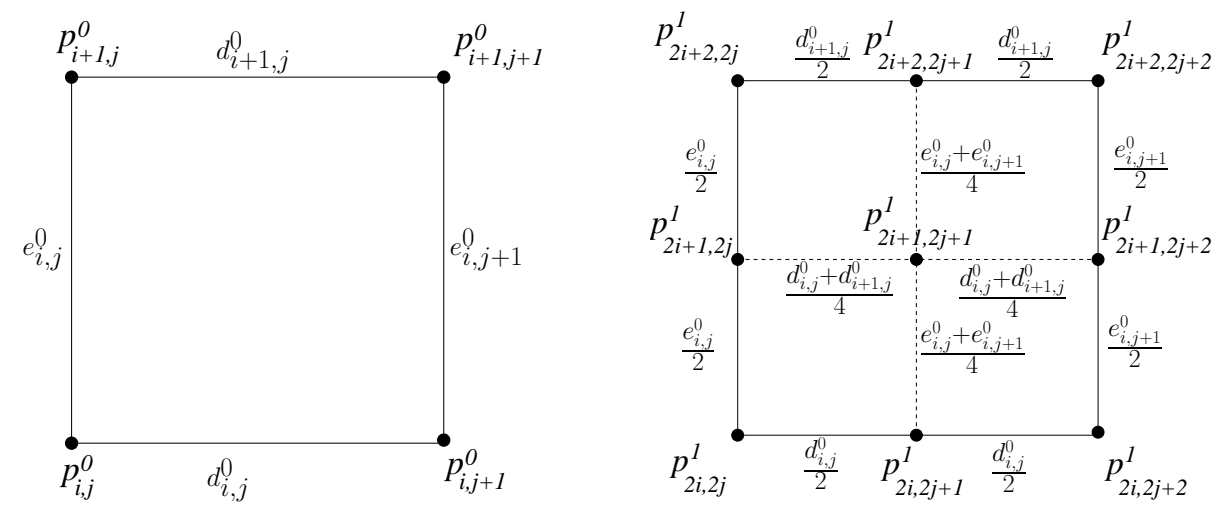

Figure 4: Knot intervals on one face of $\mathcal{M}^{0}$ (left) and on the corresponding refined face of $\mathcal{M}^{1}$ (right).

knot intervals on the opposite new edges of the refined face. In this way, for the illustrated face, the $k$ th level knot intervals are expressed by the updating formulas

$$
\begin{aligned}
d_{2 i, 2 j}^{k+1}=d_{2 i, 2 j+1}^{k+1}:=\frac{1}{2} d_{i, j}^{k}, & d_{2 i+1,2 j}^{k+1}=d_{2 i+1,2 j+1}^{k+1}:=\frac{1}{4}\left(d_{i, j}^{k}+d_{i+1, j}^{k}\right), \\
e_{2 i, 2 j}^{k+1}=e_{2 i+1,2 j}^{k+1}:=\frac{1}{2} e_{i, j}^{k}, & e_{2 i, 2 j+1}^{k+1}=e_{2 i+1,2 j+1}^{k+1}:=\frac{1}{4}\left(e_{i, j}^{k}+e_{i, j+1}^{k}\right) .
\end{aligned}
$$

After a global rescaling by 2 of the knot intervals (which does not change the next level coefficients of the scheme being the basis functions in (3) scaling invariant), we get the simplified expressions

$$
\begin{array}{ll}
d_{2 i, 2 j}^{k+1}=d_{2 i, 2 j+1}^{k+1}=d_{i, j}^{k}, & d_{2 i+1,2 j}^{k+1}=d_{2 i+1,2 j+1}^{k+1}=\frac{1}{2}\left(d_{i, j}^{k}+d_{i+1, j}^{k}\right), \\
e_{2 i, 2 j}^{k+1}=e_{2 i+1,2 j}^{k+1}=e_{i, j}^{k}, & e_{2 i, 2 j+1}^{k+1}=e_{2 i+1,2 j+1}^{k+1}=\frac{1}{2}\left(e_{i, j}^{k}+e_{i, j+1}^{k}\right) .
\end{array}
$$

Remark 1. If we restrict our attention to a single face of $\mathcal{M}^{0}$, for instance the one in Fig.4, we observe that after $k \geqslant 1$ refinements its parameters can be written in terms of the initial parameters via

$$
\begin{aligned}
& d_{2^{k} i+r, 2^{k} j+\ell}^{k}=d_{i, j}^{0}+r \frac{d_{i+1, j}^{0}-d_{i, j}^{0}}{2^{k}}, \quad r=0, \ldots, 2^{k}, \ell=0, \ldots, 2^{k}-1, \\
& e_{2^{k} i+r, 2^{k} j+\ell}^{k}=e_{i, j}^{0}+\ell \frac{e_{i, j+1}^{0}-e_{i, j}^{0}}{2^{k}}, \quad r=0, \ldots, 2^{k}-1, \ell=0, \ldots, 2^{k} .
\end{aligned}
$$

The iterated application of the knot intervals updating method generates a particular parameterization that we call augmented. If we focus on one refined initial face we observe that after $k$ subdivisions the knot intervals on the inserted edges are equal along each section polyline inside the face, as illustrated in Figure 5 (left), whereas they change when moving to the neighboring initial faces. This allows us to make the following observation which is crucial to analyze the continuity and smoothness of NULISS.

Remark 2. We notice that, since the coefficients of the scheme are invariant under uniform scaling of the knot intervals, in the interior of each initial face the knot intervals can be equivalently thought as uniform in both grid directions (Fig. 5(right)). As a consequence, all the points of the grid delimited in bold in figure are actually generated by the tensor product of the reference scheme with uniform parameterization. 

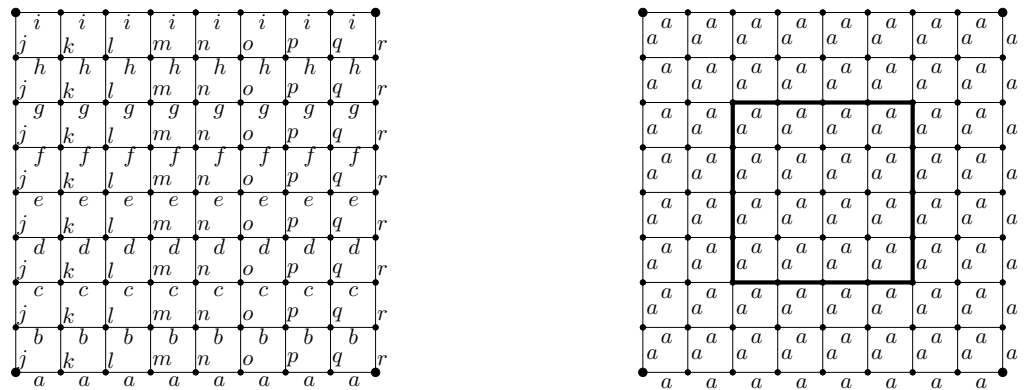

Figure 5: Knot intervals configuration on the edges of the face illustrated in Fig. 4 after 3 subdivisions (left) and region where the uniform tensor product scheme has been applied (right).

\subsection{The non-uniform tensor product scheme: a subcase of NULISS}

We consider the special case in which the initial parameterization for NULISS is chosen as the cartesian product of two non-uniform sequences of knot intervals $\mathcal{D}^{0}=\left\{d_{i}^{0}\right\}_{i}$ and $\mathcal{E}^{0}=\left\{e_{j}^{0}\right\}_{j}$ in the two grid directions. In this case, according to the updating rule (10), the refined mesh $\mathcal{M}^{k}$ is associated with the cartesian product of two non-uniform parameterizations

$$
\begin{aligned}
\mathcal{D}^{k} & =\{\underbrace{d_{0}^{0}, \ldots, d_{0}^{0}}_{2^{k}}, \underbrace{d_{1}^{0}, \ldots, d_{1}^{0}}_{2^{k}}, \underbrace{d_{2}^{0}, \ldots, d_{2}^{0}}_{2^{k}}, \ldots\}, \\
\mathcal{E}^{k} & =\{\underbrace{e_{0}^{0}, \ldots, e_{0}^{0}}_{2^{k}}, \underbrace{e_{1}^{0}, \ldots, e_{1}^{0}}_{2^{k}}, \underbrace{e_{2}^{0}, \ldots, e_{2}^{0}}_{2^{k}}, \ldots\},
\end{aligned}
$$

and thus NULISS becomes a non-uniform tensor product scheme.

Moreover, it can be easily observed that the coefficients of NULISS in (7) and (9) are independent of the level $k$ and thus generate a stationary subdivision operator mapping the points of the control mesh $\mathcal{M}^{k}$ into points of $\mathcal{M}^{k+1}$ for any $k \geqslant 0$. The following result derives straightforwardly from the assumption that the reference scheme is $C^{1}$.

Theorem 1. The NULISS scheme with cartesian product parameterization generates $C^{1}$ continuous limit surfaces.

\section{Convergence and smoothness analysis}

In this section we analyze the convergence and smoothness properties of NULISS. In particular, we prove that, assumed that the reference scheme satisfies the requirements stated in Section 2, NULISS generates $C^{1}$-continuous limit surfaces independently of the initial parameters configuration.

The approach we follow strongly relies on the limit behavior of the augmented parameterization as well as on the fact that NULISS can be also interpreted as a scheme generating sequences of curve networks. For a better understanding of these two key ingredients at the basis of our analysis, in the next section we describe in detail the structure of $\mathcal{M}^{k}$ and its behavior in the limit.

\subsection{The structure of $\mathcal{M}^{k}$ and its limit behavior}

The insertion rule for edge points in (7) implies that each section polyline, either of the initial mesh $\mathcal{M}^{0}$ or of any of the refined meshes $\mathcal{M}^{k}$, is refined independently from the underlying control mesh, namely the points inserted along the polyline depend on the polyline vertices only. As a consequence NULISS can be also viewed as an interpolatory subdivision scheme for curve networks. Specifically, the edges of the control mesh $\mathcal{M}^{k}$ define a polyline network that, when subdivided, generates in the limit a curve network, hereinafter denoted by $\mathcal{F}^{k}$. Being the scheme interpolatory, the curves in $\mathcal{F}^{k}$ intersect at the vertices of $\mathcal{M}^{k}$ and $\mathcal{F}^{0} \subset \mathcal{F}^{1} \subset \ldots \mathcal{F}^{k}, \forall k$. Figure 6 illustrates the situation. 
Obviously, if NULISS admits a limit surface, then such surface interpolates not only the vertices of each $\mathcal{M}^{k}$, but all the curves of each network $\mathcal{F}^{k}, \forall k \geqslant 0$.

Now, if we focus our attention on a subregion of $\mathcal{M}^{k}$ corresponding to a refined face of $\mathcal{M}^{0}$, as already observed in Remark 2, we have that the parameters on its edges are equal and thus can be thought as scaled to uniform; so in the innermost area of each refined initial face NULISS is nothing but the tensor product of the reference scheme, which is $C^{1}$. Extending the reasoning to the whole mesh $\mathcal{M}^{k}$ we observe that there exists a 4-wide strip of faces (see first illustration in Figure 7), surrounding the boundary of the refined faces of $\mathcal{M}^{0}$, where the parameters cannot be interpreted as uniform and thus the parameterization is actually augmented. As illustrated in Figure 7, during the subdivision process the augmented area shrinks at each iteration, while the tensor product area progressively extends to the refined section polylines of $\mathcal{M}^{0}$, which in the limit become the curves of $\mathcal{F}^{0}$. As a consequence the limit surface of NULISS is $C^{1}$ everywhere, except at points belonging to $\mathcal{F}^{0}$. Based on this reasoning, the limit surface is everywhere continuous if the $C^{1}$ limit surface patches generated by NULISS in the innermost area of each refined initial face join with continuity along the curves of $\mathcal{F}^{0}$. Moreover, it is even $C^{1}$ if such patches join with $C^{1}$ continuity across the curves of $\mathcal{F}^{0}$.

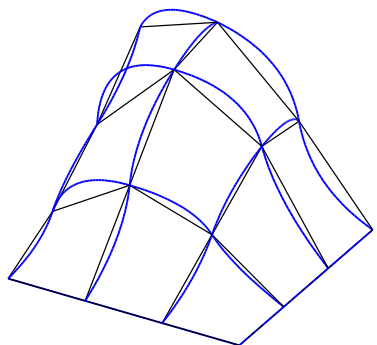

(a) $\mathcal{M}^{0}$ and $\mathcal{F}^{0}$ (blue)

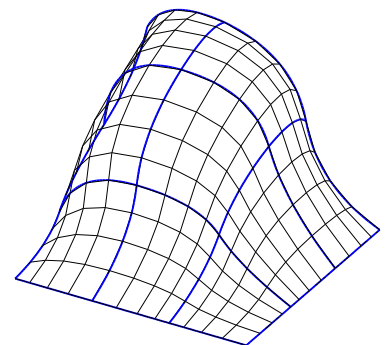

(b) $\mathcal{M}^{2}$ and $\mathcal{F}^{0}$ (blue)

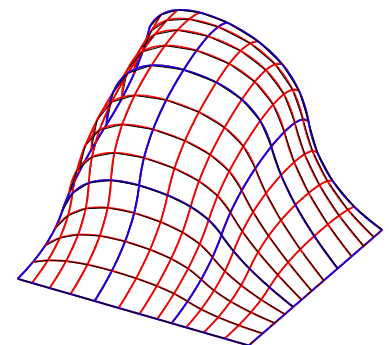

(c) $\mathcal{M}^{2}, \mathcal{F}^{0}$ (blue) and $\mathcal{F}^{2}$ (blue and red)

Figure 6: Meshes $\mathcal{M}^{k}$ and curve networks $\mathcal{F}^{k}$ generated by NULISS.
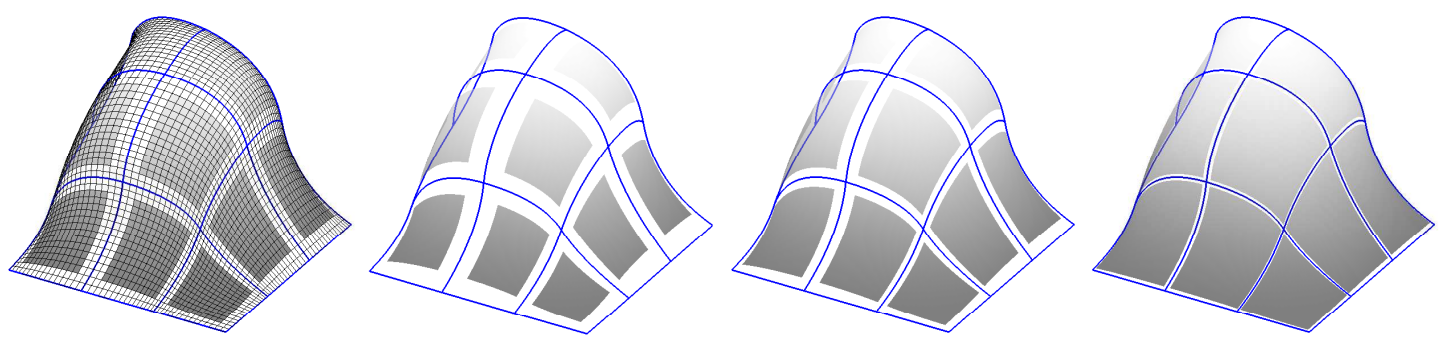

Figure 7: Curve network $\mathcal{F}^{0}$ and $C^{1}$ tensor product limit surface patches at subsequent refinement steps. The tensor product area progressively extends to the boundary of the refined faces of $\mathcal{M}^{0}$, which in the limit is $\mathcal{F}^{0}$, while the augmented area shrinks.

\subsection{Continuity and smoothness analysis of NULISS}

In this section we firstly prove that, at any arbitrary point of $\mathcal{F}^{0}$, the limit surface is continuous and then even $C^{1}$. We start proving continuity. To this aim we observe that it is sufficient to show that the limit surface is continuous at any dyadic point, i.e. at any point of $\mathcal{M}^{k} \cap \mathcal{F}^{0}, \forall k \geqslant 0$. In fact supposed $P$ is an arbitrary point of $\mathcal{F}^{0}$, and being any curve in $\mathcal{F}^{0}$ continuous, then $P$ is the limit of a sequence of dyadic points belonging to $\mathcal{F}^{0}$. So, if the limit surface is continuous at any point of the sequence, it is certainly continuous also at $P$. Based on this observation, from this point onward we focus on a dyadic point, hereinafter denoted by $V$, belonging to $\mathcal{M}^{k} \cap \mathcal{F}^{0}, \forall k \geqslant 0$ and we prove that the NULISS limit surface is continuous at such point. To analyze the continuity of NULISS we study the asymptotical 
behavior of the augmented parameterization in a suitable neighborhood of $V$ and show that the local matrix operator of NULISS is asymptotically equivalent to the local matrix operator of a bivariate convergent subdivision scheme. This implies that NULISS is also convergent [18]. In particular we compare the local matrix operator of NULISS with that of the tensor product scheme described in Section 3.2, defined on the parameterization of the two section polylines of $\mathcal{M}^{k} \cap \mathcal{F}^{0}$ intersecting at $V$.

We also observe that NULISS has support of width 6 in each grid direction. This property is a direct consequence of the well known fact that the 4-point reference scheme has support of width 6 . Thus the parameters that influence the subdivision process are limited to the 3-neighborhood of $V$.

Assuming the point $V$ is created at subdivision level $K$, then $V \in \mathcal{M}^{k} \cap \mathcal{F}^{0}, \forall k \geqslant K$. Before starting the analysis, we make a preliminary observation that allows us to significantly simplify the proof. We consider the two cases

1. $K=0 \Rightarrow V \in \mathcal{M}^{0}$ (Fig. 8-left);

2. $K>0 \Rightarrow V \in \mathcal{M}^{K} \backslash \mathcal{M}^{0} \cap \mathcal{F}^{0}$ (Fig. 8-right).
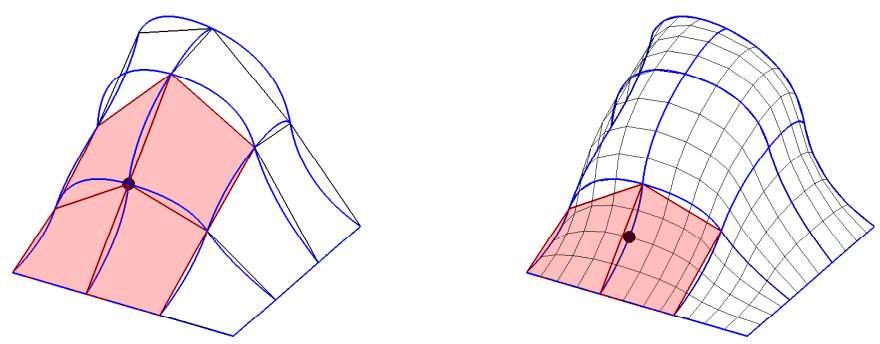

Figure 8: Illustration of the two possible cases of the point $V$ depicted by a black bullet.

In case 1., after $k$ subdivision steps, the parameters on the edges of such region depend on the initial parameters of the four faces of $\mathcal{M}^{0}$ meeting at $V$ (Fig.8-left) and they are augmented in both grid directions. In case 2., the parameters depend on those of the two faces of $\mathcal{M}^{0}$ having in common the edge from which $V$ is generated by the iterative refinement (Fig.8-right); in such situation the parameters can be thought as uniform in the direction parallel to the edge and augmented across it. Thus this second case falls into the first, and in the following we focus the analysis on case 1 . only.

Without loss of generality, we can assume that the point $V$ around which we want to prove the convergence, is the initial vertex $p_{i, j}^{0}$ and, being the scheme interpolatory, $V=p_{i, j}^{0}=p_{2^{k} i 2^{k} j}^{k}, \forall k>0$. Figure 9 represents the face containing $V$ in the top-right quadrant (Fig.9(a)) and the corresponding $k$ times refined face (Fig.9 (b)).

We consider now the sequences of parameters of the two initial section polylines intersecting at $V$

$$
\mathcal{D}_{i}^{0}=\left\{d_{i, j}^{0}\right\}_{j}, \quad \text { and } \quad \mathcal{E}_{j}^{0}=\left\{e_{i, j}^{0}\right\}_{i},
$$

and the non-uniform tensor product scheme defined from the cartesian product of them as described in Section 3.2.

Definition 1. We denote by $S_{\mathcal{D E}}(V)$ the tensor product scheme obtained from NULISS when the initial parameterization is chosen as the cartesian product of the parameterizations of the two initial section polylines intersecting at V.

In the following we prove that the local matrix operators of NULISS and $S_{\mathcal{D E}}(V)$ are asymptotically equivalent. To this aim we start by introducing preliminary results which strongly rely on the properties of the basis functions from which the coefficients of the reference scheme are derived according to (4).

Lemma 1. For any $k \geqslant 1$, let $\delta=\left[u+2^{-k} C, v+2^{-k} D, v+2^{-k} D\right]$, with $u, v>0, C, D \in \mathbb{R}$, be a triple of knot intervals associated to knots $x_{j}, j=0, \ldots, 3$ with $x_{0}=0$. Let also $J=\left[0, \max \left(u+2 v, u+2 v+\frac{C+2 D}{2}\right)\right]$ be the interval containing $x_{j}, j=0, \ldots, 3$, for all $k \geqslant 1$. Then for any $k \geqslant 1$ and $\ell=0, \ldots, 3$, 
(a) $\left|\psi_{\ell}^{\delta}(z)\right| \leqslant A$ for all $z \in J$, with $A$ a generic constant independent of $k$;

(b) $\psi_{\ell}^{\delta}$ is Lipschitz continuous on J, with a constant L independent of $k$.

Proof: We prove the two statements when $C, D \geqslant 0$ since for either $C, D$ or both negative we can proceed analogously. Since the cardinal basis functions are invariant under a uniform scaling of the knot intervals, we can assume $\delta=$ $[x, 1,1]$, with $x \in I:=\left[\min \left(\frac{u}{v}, \frac{2 u+C}{2 v+D}\right), \max \left(\frac{u}{v}, \frac{2 u+C}{2 v+D}\right)\right]$. In this way, varying $x$ in $I$, we obtain all possible triples of knot intervals for $k \geqslant 1$. Then, for all $\ell=0, \cdots, 3$, we consider the continuous function $f_{\ell}(x, z):=\psi_{\ell}^{\delta_{(}}(z)$ on $I \times J$.

(a) From the continuity of $f_{\ell}(x, z)$ we can conclude that $A=\max _{(x, z) \in I \times J}\left|f_{\ell}(x, z)\right|$, which is independent of $k$.

(b) Due to property (ii) of the cardinal basis functions we have that, for all $\ell=0, \ldots, 3, f_{\ell}(x, z)$ is differentiable with respect to $z$ and its partial derivative $\frac{\partial}{\partial z} f_{\ell}(x, z)$ is continuous on $I \times J$. Thus we can conclude that $L=\max _{(x, z) \in I \times J}\left|\frac{\partial}{\partial z} f_{\ell}(x, z)\right|$, which is independent of $k$.

Lemma 2. For any $k \geqslant 1$, let $\delta=\left[u+2^{-k} C, v+2^{-k} D, v+2^{-k} D\right]$, with $u, v>0, C, D \in \mathbb{R}$, be a triple of knot intervals associated to knots $x_{j}, j=0, \ldots, 3$ with $x_{0}=0$. Let $\hat{\boldsymbol{\delta}}=[u, v, v]$ be a triple of knot intervals associated to knots $\hat{x}_{j}$, $j=0, \ldots, 3$ with $\hat{x}_{0}=0$. Denote also $m_{\boldsymbol{\delta}}=u+\frac{v}{2}+\frac{2 C+D}{2^{k+1}}$ and $m_{\hat{\delta}}=u+\frac{v}{2}$. Then for any $k \geqslant \vec{k}$, with $\bar{k}$ a suitable positive integer,

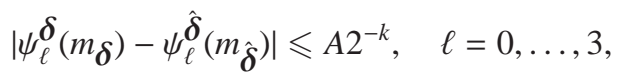

with A a generic constant independent of $k$.

Proof: Let us start assuming that $C$ and $D$ are non-negative values. From the property of cardinality of the fundamental functions $\psi_{\ell}^{\delta}$ on $x_{j}, j=0, \ldots, 3$, we can write

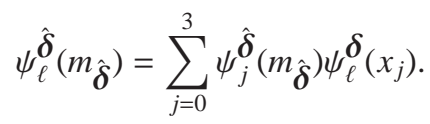

Moreover, since the fundamental functions $\psi_{\ell}^{\hat{\delta}}$ reproduce polynomials of degree $\rho$ and $\psi_{\ell}^{\delta}$ is a polynomial of degree $\rho$ on $\left[x_{1}, x_{2}\right]$, by property (iii) of the fundamental functions we get

$$
\psi_{\ell}^{\boldsymbol{\delta}_{(}}\left(m_{\boldsymbol{\delta}}\right)=\sum_{j=0}^{3} \psi_{j}^{\hat{\boldsymbol{\delta}}_{(}}\left(m_{\boldsymbol{\delta}}\right) \psi_{\ell} \boldsymbol{\delta}_{\left(\hat{x}_{j}\right)}
$$

For $k \geqslant \vec{k}$, with $\bar{k}$ a suitable positive integer depending on $u, v, C, D$, we have $m_{\hat{\delta}}, m_{\boldsymbol{\delta}} \in I_{1}=\left[u+2^{-\bar{k}} C, u+v\right]$. Now, let $I_{2}=\left[0, u+2 v+\frac{C+2 D}{2}\right]$ be the smallest interval containing the knots $\hat{x}_{j}$ and $x_{j}, j=0, \ldots, 3$ for all $k \geqslant 1$. For $\left(z_{1}, z_{2}\right) \in I_{1} \times I_{2}$, we define the bivariate function $g\left(z_{1}, z_{2}\right):=\psi_{j}^{\hat{\delta}_{(}}\left(z_{1}\right) \psi_{\ell}^{\boldsymbol{\delta}_{(}}\left(z_{2}\right)$. Since $\psi_{j}^{\hat{\boldsymbol{\delta}}}$ and $\psi_{\ell}^{\boldsymbol{\delta}}$ are Lipschitz continuous, the function $g$ is also Lipschitz continuous with constant

$$
\bar{L}=L \max _{z_{1} \in I_{1}}\left|\psi_{j}^{\hat{\boldsymbol{\delta}}_{(}}\left(z_{1}\right)\right|+L_{\hat{\boldsymbol{\delta}}} \max _{z_{2} \in I_{2}}\left|\psi_{\ell}^{\delta}\left(z_{2}\right)\right|
$$

where $L_{\hat{\boldsymbol{\delta}}}$ and $L$ are the Lipschitz constants of $\psi_{j}^{\hat{\boldsymbol{\delta}}}$ and $\psi_{\ell}^{\delta}$, respectively. We observe that all the contributions in $\bar{L}$ are independent of $k$ : this is trivial for $L_{\hat{\delta}}$, $\max _{z_{1} \in I_{1}}\left|\psi_{j}^{\delta_{(}}\left(z_{1}\right)\right|$ and derives from Lemma 1 (a) and (b) for $\max _{z_{2} \in I_{2}} \mid \psi_{\ell} \delta_{\left(z_{2}\right) \mid \text { and }}$ $L$, respectively. Thus,

$$
\begin{gathered}
\left|\psi_{\ell}^{\boldsymbol{\delta}}\left(m_{\boldsymbol{\delta}}\right)-\psi_{\ell}^{\hat{\boldsymbol{\delta}}_{(}}\left(m_{\hat{\boldsymbol{\delta}}}\right)\right|=\left|\sum_{j=0}^{3}\left(\psi_{j}^{\boldsymbol{\delta}_{(}}\left(m_{\boldsymbol{\delta}}\right) \psi_{\ell}^{\boldsymbol{\delta}}\left(\hat{x}_{j}\right)-\psi_{j}^{\boldsymbol{\boldsymbol { \delta }}_{(}}\left(m_{\hat{\boldsymbol{\delta}}}\right) \psi_{\ell}^{\boldsymbol{\delta}}\left(x_{j}\right)\right)\right| \leqslant \sum_{j=0}^{3}\left|g\left(m_{\boldsymbol{\delta}}, \hat{x}_{j}\right)-g\left(m_{\hat{\boldsymbol{\delta}}}, x_{j}\right)\right| \\
\leqslant 4 \bar{L} \max _{j=0, \ldots, 3}\left\{\left|m_{\boldsymbol{\delta}}-m_{\hat{\boldsymbol{\delta}}^{1}}\right|, \hat{x}_{j}-x_{j} \mid\right\} .
\end{gathered}
$$


Considering that

$$
\max _{j=0, \ldots, 3}\left\{\left|m_{\delta}-m_{\hat{\boldsymbol{\delta}}}\right|,\left|\hat{x}_{j}-x_{j}\right|\right\}=\max \left\{\frac{2 C+D}{2^{k+1}}, \frac{C}{2^{k}}, \frac{C+D}{2^{k}}, \frac{C+2 D}{2^{k}}\right\}=\frac{C+2 D}{2^{k}}
$$

we have

$$
\left|\psi_{\ell}^{\boldsymbol{\delta}_{(}}\left(m_{\boldsymbol{\delta}}\right)-\psi_{\ell}^{\hat{\boldsymbol{\delta}}_{(}}\left(m_{\hat{\boldsymbol{\delta}}}\right)\right| \leqslant 2^{-k} 4 \bar{L}(C+2 D)=A 2^{-k},
$$

for a generic constant $A$ independent of $k$. This proves our statement for $C$ and $D$ non-negative. If either $C, D$ or both are negative, we can repeat the proof in a similar way.

Exploiting the above lemmas and the asymptotical behavior of the augmented parameterization we can now formulate the following result.

Theorem 2. At any dyadic point $V$ the local matrix operator of NULISS is asymptotically equivalent to the local matrix operator of the tensor product scheme $S_{\mathcal{D E}}(V)$.

Proof: We formalize the subdivision process of NULISS in the neighborhood of $V$ in terms of the local subdivision matrix $M_{k}$ mapping the vertices of the $k$ th level mesh $\mathcal{M}^{k}$ into those of $\mathcal{M}^{k+1}$ via

$$
P^{k+1}=M_{k} P^{k}
$$

The non-zero entries of each row of $M_{k}$ are the coefficients of the vertex, edge and face point refinement equations of NULISS and depend on the local parameterization associated with $k$-level edges. Since the reference scheme has support width 6 , it is easy to see that $M_{k}$ has 49 rows and columns. We introduce the following local notation (see Figure 9(g)-(h))

$$
p_{r, s}:=p_{2^{k} i+r, 2^{k} j+s}^{k}, \quad \boldsymbol{\delta}_{r, s}:=\delta_{2^{k} i+r, 2^{k} j+s}^{k}, \quad \boldsymbol{\epsilon}_{r, s}:=\boldsymbol{\epsilon}_{2^{k} i+r, 2^{k} j+s}^{k}, \quad r, s=-3, \ldots, 3 .
$$

The points $p_{r, s}, r, s=-3, \ldots, 3$ are the entries of $P^{k}$ to the right hand side of (14), and they constitute the submesh of the three rings of faces of $\mathcal{M}^{k}$ around $V$. We denote this submesh, illustrated in color red in Figure 9(c),(e),(f), by $\mathcal{G}^{k}(V)$. Similarly $\boldsymbol{\delta}_{r, s}$ and $\boldsymbol{\epsilon}_{r, s}$ are the parameters on the edges of $\mathcal{G}^{k}(V)$ that contribute to the entries of $M_{k}$. The points $P^{k+1}$ to the left of (14) are the vertices of $G^{k+1}(V)$ (Figure $9(\mathrm{~d})$ in color red).

We also denote by $M$ the subdivision matrix having as rows the coefficients of vertex, edge and face points of $S_{\mathcal{D E}}(V)$. The local matrix operators of NULISS and $S_{\mathcal{D E}}(V)$ are asymptotically equivalent if $\sum_{k \in \mathbb{Z}^{+}}\left\|M_{k}-M\right\|_{\infty}<\infty$ [18]. Being $\left\|M_{k}-M\right\|_{\infty}=\max _{i} \sum_{j}\left|m_{i, j}^{k}-m_{i, j}\right|$, we prove that,

$$
\sum_{j}\left|m_{i, j}^{k}-m_{i, j}\right| \leqslant A 2^{-k}, \quad \forall i,
$$

with $A$ a generic constant independent of $k$.

In the following we thus focus our attention on the rows of $M_{k}$ and $M$ corresponding to vertex, edge and face points and show that relation (16) holds for each of them. By symmetry of the subdivision rules we can limit ourselves to consider only rows $i$ corresponding to points of $\mathcal{G}^{k+1}(V)$ inserted in the top-right quadrant (Figure 9(e)-(f)).

- Being the two schemes interpolatory, if the $i$ th row represents a vertex point, nothing needs to be proved.

- Suppose now that the $i$ th row of $M_{k}-M$ corresponds to an edge point $E$ (Figure 9(e)). We can express any edge point of NULISS in the horizontal direction and in the top-right quadrant by the formula

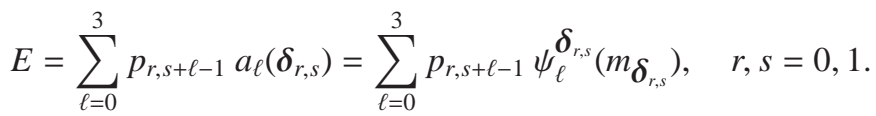




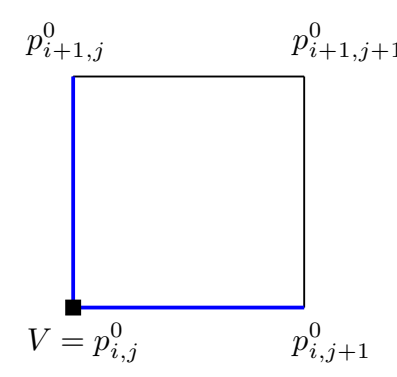

(a) face of $\mathcal{M}^{0}$ containing $V$

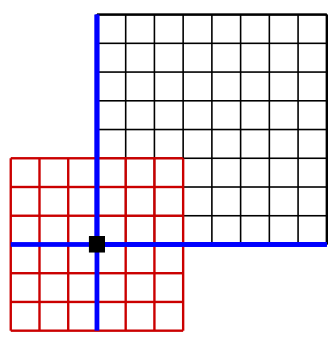

(c) local grid $\mathcal{G}^{k}(V) \subset \mathcal{M}^{k}$

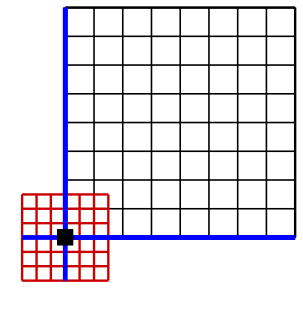

(d) local grid $\mathcal{G}^{k+1}(V) \subset \mathcal{M}^{k+1}$

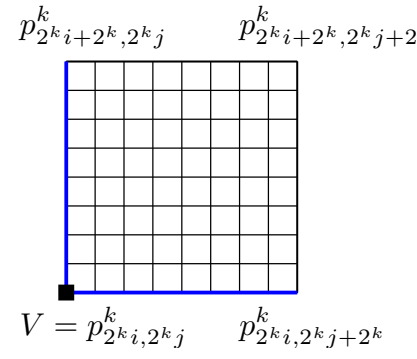

(b) face in (a) after $k$ refinements

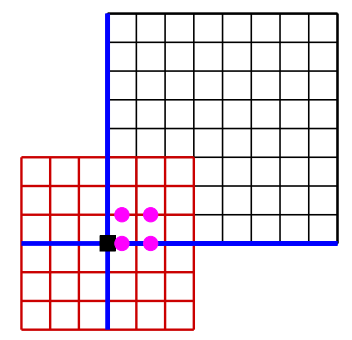

(e) edge points in one quadrant

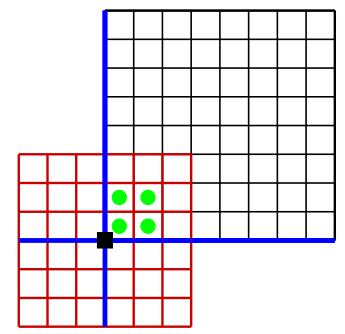

(f) face points in one quadrant

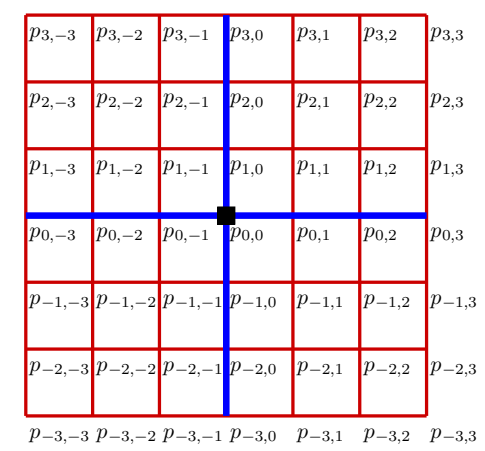

(g) points of $\mathcal{G}^{k}(V)$ with indexing in (15)

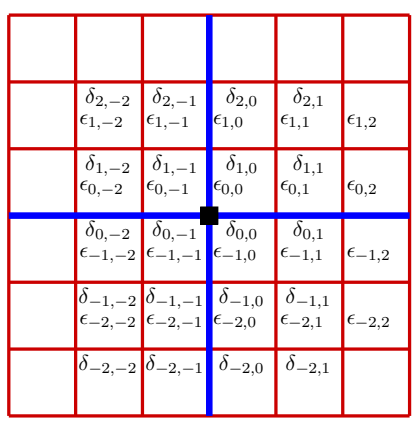

(h) parameter sequences on $\mathcal{G}^{k}(V)$ for NULISS

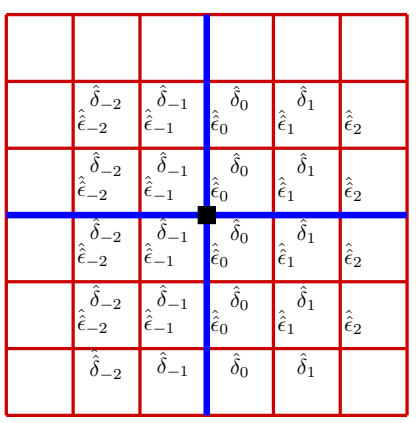

(i) parameter sequences on $\mathcal{G}^{k}(V)$ for $\mathcal{S}_{\mathcal{D E}}(V)$

Figure 9: Illustration of the local configuration of points and parameters used in the proof of Theorem 2. 
An analogous equation holds in the vertical direction. Exploiting equation (11) we can write $\boldsymbol{\delta}_{r, s}$ in dependence of the knot intervals of $\mathcal{M}^{0}$, namely for $r=0,1$,

$$
\boldsymbol{\delta}_{r, s}= \begin{cases}{\left[d_{i, j-1}^{0}+r \frac{d_{i+1, j-1}^{0}-d_{i, j-1}^{0}}{2^{k}}, d_{i, j}^{0}+r \frac{d_{i+1, j}^{0}-d_{i, j}^{0}}{2^{k}}, d_{i, j}^{0}+r \frac{d_{i+1, j}^{0}-d_{i, j}^{0}}{2^{k}}\right]} & \text { when } s=0, \\ {\left[d_{i, j}^{0}+r \frac{d_{i+1, j}^{0}-d_{i, j}^{0}}{2^{k}}, d_{i, j}^{0}+r \frac{d_{i+1, j}^{0}-d_{i, j}^{0}}{2^{k}}, d_{i, j}^{0}+r \frac{d_{i+1, j}^{0}-d_{i, j}^{0}}{2^{k}}\right]} & \text { when } s=1 .\end{cases}
$$

Furthermore we denote the edge point of $S_{\mathcal{D E}}(V)$, corresponding to the same location of $E$, by $\hat{E}$. $\hat{E}$ is defined as the linear combination (7), with coefficients obtained evaluating the basis functions $\psi_{\ell}^{\boldsymbol{\delta}_{0, s}}$ associated to the parameterization $\delta_{0, s}, s=0,1$. Notice that such parameterization is common to all the considered rows. As a consequence of the affine invariance of the basis functions the parameterization $\boldsymbol{\delta}_{0, s}$ generates the same coefficients as (Figure 9(i))

$$
\hat{\boldsymbol{\delta}}_{s}= \begin{cases}{\left[d_{i, j-1}^{0}, d_{i, j}^{0}, d_{i, j}^{0}\right]} & \text { when } s=0, \\ {\left[d_{i, j}^{0}, d_{i, j}^{0}, d_{i, j}^{0}\right]} & \text { when } s=1 .\end{cases}
$$

Thus we can write

$$
\hat{E}=\sum_{\ell=0}^{3} p_{r, s+\ell-1} a_{\ell}\left(\hat{\boldsymbol{\delta}}_{s}\right)=\sum_{\ell=0}^{3} p_{r, s+\ell-1} \psi_{\ell}^{\hat{\boldsymbol{\delta}}_{s}}\left(m_{\hat{\boldsymbol{\delta}}_{s}}\right), \quad r, s=0,1,
$$

from which we get

$$
E-\hat{E}=\sum_{\ell=0}^{3} p_{r, s+\ell-1}\left(\psi_{\ell}^{\boldsymbol{\delta}_{r, s}}\left(m_{\boldsymbol{\delta}_{r, s}}\right)-\psi_{\ell}^{\hat{\boldsymbol{\delta}}_{s}}\left(m_{\hat{\boldsymbol{\delta}}_{s}}\right)\right) .
$$

We now observe that for $k$ big enough, $\boldsymbol{\delta}_{r, s}$ in (17) and $\hat{\boldsymbol{\delta}}_{s}$ in (18) fall into the hypothesis of Lemma 2 with $u=d_{i, j-1}^{0}, v=d_{i, j}^{0}, C=r\left(d_{i+1, j-1}^{0}-d_{i, j-1}^{0}\right), D=r\left(d_{i+1, j}^{0}-d_{i, j}^{0}\right)$ and thus

$$
\left|\psi_{\ell}^{\boldsymbol{\delta}_{r, s}}\left(m_{\boldsymbol{\delta}_{r, s}}\right)-\psi_{\ell}^{\hat{\boldsymbol{\delta}}_{s}}\left(m_{\hat{\boldsymbol{\delta}}_{s}}\right)\right| \leqslant A 2^{-k}, \quad \forall \ell=0, \cdots, 3,
$$

where $A$ is a generic constant independent of $k$. Therefore, when the row $i$ identifies an edge point rule,

$$
\sum_{j}\left|m_{i, j}^{k}-m_{i, j}\right|=\sum_{\ell=0}^{3}\left|\psi_{\ell}^{\boldsymbol{\delta}_{r, s}}\left(m_{\boldsymbol{\delta}_{r, s}}\right)-\psi_{\ell}^{\hat{\boldsymbol{\delta}}_{s}}\left(m_{\hat{\boldsymbol{\delta}}_{s}}\right)\right| \leqslant A 2^{-k}
$$

which proves relation (16).

- For a face point inside the considered region (Figure 9(f)) we recall that the coefficients of NULISS depend on local averages of knot intervals in the two grid directions as in (8). We introduce the following notation, similar to $(17)$,

$$
\boldsymbol{\Delta}_{r, s}:=\boldsymbol{\Delta}_{2^{k} i+r, 2^{k} j+s}^{k}, \quad \boldsymbol{E}_{r, s}:=\boldsymbol{E}_{2^{k} i+r, 2^{k} j+s}^{k}, \quad r, s=-2 \ldots 2 .
$$

Also, in analogy to (18), we set $\hat{\boldsymbol{\Delta}}_{s}:=\boldsymbol{\Delta}_{0, s}$ and $\hat{\boldsymbol{E}}_{r}:=\boldsymbol{E}_{r, 0}$. Thus

$$
\begin{aligned}
& F-\hat{F}=\sum_{h=0}^{3} \sum_{\ell=0}^{3} p_{r+h-1, s+\ell-1} a_{h}\left(\boldsymbol{\Delta}_{r, s}\right) a_{\ell}\left(\boldsymbol{E}_{r, s}\right)-\sum_{h=0}^{3} \sum_{\ell=0}^{3} p_{r+h-1, s+\ell-1} a_{h}\left(\hat{\boldsymbol{\Delta}}_{s}\right) a_{\ell}\left(\hat{\boldsymbol{E}}_{r}\right)
\end{aligned}
$$

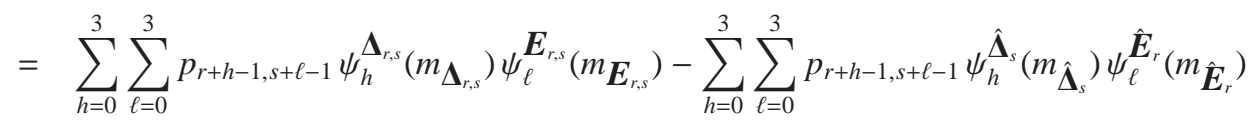

$$
\begin{aligned}
& =\sum_{h=0}^{3}\left\{\sum_{\ell=0}^{3} p_{r+h-1, s+\ell-1}\left[\psi_{\ell}^{\boldsymbol{E}_{r, s}}\left(m_{\boldsymbol{E}_{r, s}}\right)\left(\psi_{h}^{\boldsymbol{\Delta}_{r, s}}\left(m_{\boldsymbol{\Delta}_{r, s}}\right)-\psi_{h}^{\hat{\boldsymbol{\Delta}}_{s}}\left(m_{\hat{\boldsymbol{\Delta}}_{s}}\right)\right)-\psi_{h}^{\hat{\boldsymbol{\Delta}}_{s}}\left(m_{\hat{\boldsymbol{\Delta}}_{s}}\right)\left(\psi_{\ell}^{\hat{\boldsymbol{E}}_{r}}\left(m_{\hat{\boldsymbol{E}}_{r}}\right)-\psi_{\ell}^{\boldsymbol{E}_{r, s}}\left(m_{\boldsymbol{E}_{r, s}}\right)\right)\right]\right\} .
\end{aligned}
$$


As above, for a sufficiently large $k$, we can show that $\boldsymbol{\Delta}_{r, s}$ and $\hat{\boldsymbol{\Delta}}_{s}, \boldsymbol{E}_{r, s}$ and $\hat{\boldsymbol{E}}_{r}$ satisfy the hypothesis of Lemma 2. Therefore, when $i$ identifies a face point rule,

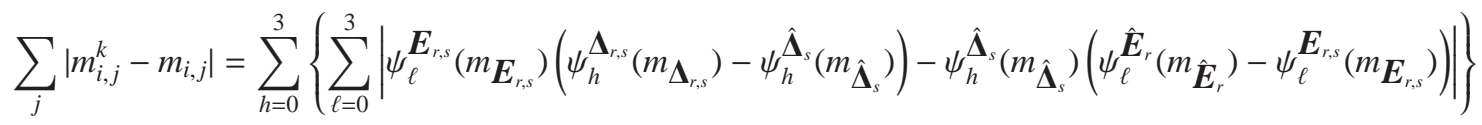

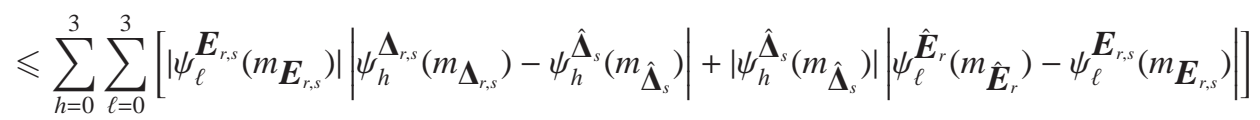

$$
\begin{aligned}
& \leqslant 2^{-k} A \sum_{h=0}^{3} \sum_{\ell=0}^{3}\left(\left|\psi_{\ell}^{\boldsymbol{E}_{r, s}}\left(m_{\boldsymbol{E}_{r, s}}\right)\right|+\left|\psi_{h}^{\hat{\boldsymbol{\Delta}}_{s}}\left(m_{\hat{\boldsymbol{\Delta}}_{s}}\right)\right|\right) \\
& \leqslant 2^{-k} A
\end{aligned}
$$

The last inequality follows from Lemma 1(a) and shows that (16) holds. This concludes the proof.

Corollary 1. NULISS is convergent and generates $C^{0}$-continuous limit surfaces.

The previous result naturally implies the following

Theorem 3. NULISS generates $C^{1}$-continuous limit surfaces.

Proof: As already observed in Section 4.1, $C^{1}$ smoothness of the limit surface in the inner region of each face of $\mathcal{M}^{0}$ is trivially established considering the limit behavior of the augmented parameterization.

We recall also that the subdivision process generates a sequence of $C^{1}$ curve networks $\left\{\mathcal{F}^{k}\right\}_{k \geqslant 0}$, where each member of the sequence is the curve network defined by the limit curves associated to the polylines of $\mathcal{M}^{k}$. Thus, any dyadic point $V \in \mathcal{M}^{k} \cap \mathcal{F}^{0}$ is the intersection of two $C^{1}$ curves of $\mathcal{F}^{k}$ in the two independent grid directions, which implies that first partial derivatives of the surface are continuous at $V$.

At an arbitrary (non dyadic) point of $\mathcal{F}^{0}$, the first derivative of the limit surface is certainly continuous in the direction of the curve of $\mathcal{F}^{0}$ containing the point. Such curve is the boundary of two $C^{1}$ patches of the continuous limit surface: so, in the transversal direction, the derivative varies continuously anywhere inside the patches and, taking into account that it is also continuous across the boundary at any dyadic point, we can conclude that it must necessarily be continuous everywhere.

\section{Numerical examples}

We conclude by presenting some numerical experimentation about NULISS in order to show the quality of NULISS surfaces and compare it with uniform and non-uniform tensor product interpolation methods.

The surfaces shown in the following figures are generated using NULISS with reference scheme in (6), where the initial knot intervals have been computed via the centripetal parameterization and, in the case of open initial meshes, exploiting linear extrapolation along the cross-boundary direction.

We start with two simple, yet effective examples. To produce the examples in Figure 10 we modified the regular torus mesh, so as to obtain an initial mesh whose section polylines have corresponding edges of remarkably different lengths (Fig. 10 (a)). In such situation, the uniform parameterization introduces a significant distortion with respect to the parameterization of the individual initial section polylines. As a consequence, an unwanted artifact appears in the limit surface of the uniform scheme (Fig. 10 (b) and (d)), which is not present in the NULISS surfaces generated from the same initial meshes (Fig. 10 (c) and (e)). The kind of artifact highlighted by this example is particularly evident if we look at one section curve of the uniform and NULISS limit surfaces, as illustrated in Fig. 11 for the upper-row mesh (Fig.10 (a)). While in the uniform case each section curve has the same parameterization in both directions, in the latter one each section curve maintains its own (centripetal) parameterization. 


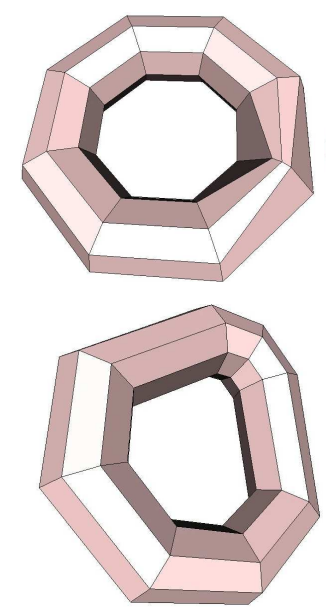

(a)Initial mesh
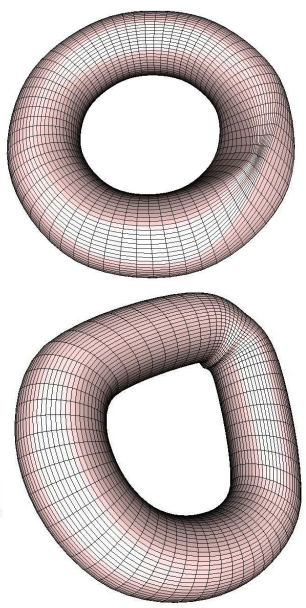

(b) Uniform, 3 steps
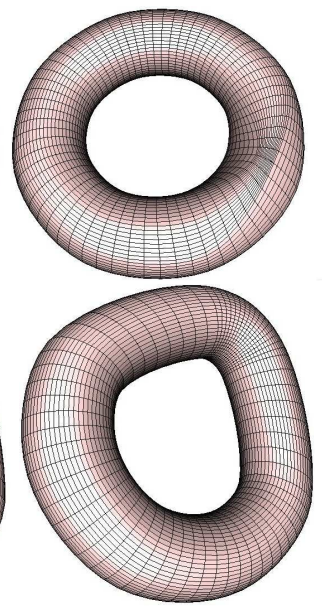

(c) NULISS, 3 steps

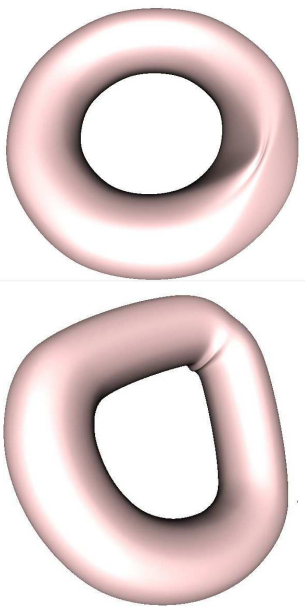

(d) Uniform limit
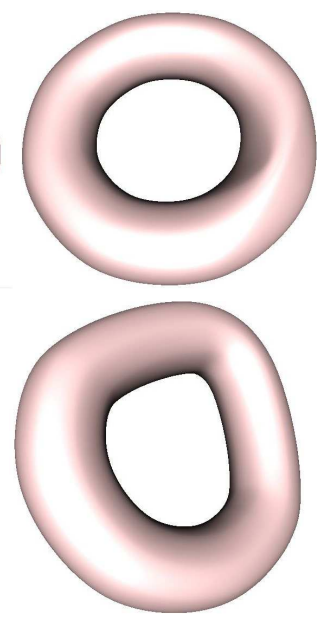

(e) NULISS limit

Figure 10: Comparison between uniform tensor product bicubic spline surface and NULISS.
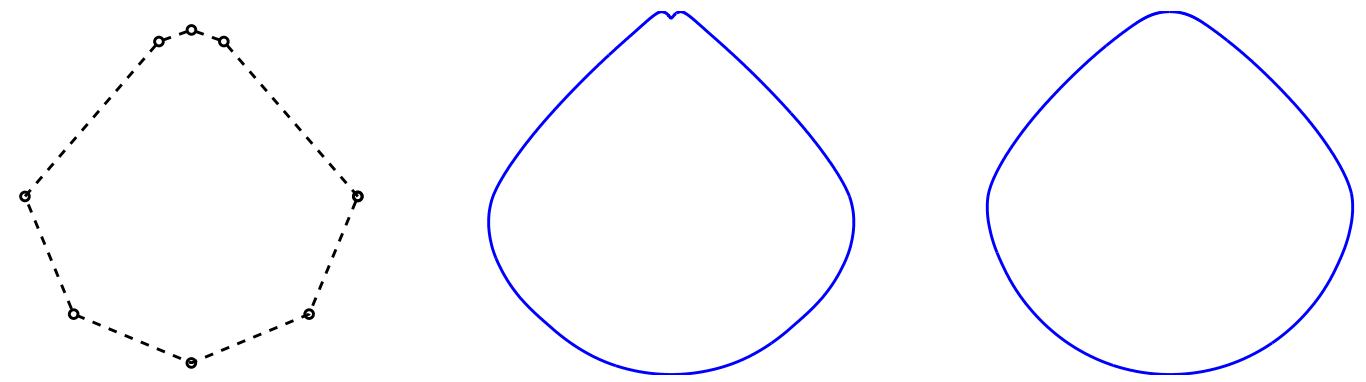

Figure 11: Section polyline of the initial mesh on the top row of Figure 10 (a) ; corresponding section curve of the uniform subdivision limit surface (d) and of NULISS (e).

To give a general idea of the quality of the surfaces, we provide in Figure 12 the results that we got by applying NULISS to a variety of initial meshes. The lamp mesh is obtained by revolution of a profile polyline with extremely short and long edges, and thus it is essentially uniform in one grid direction and highly non-uniform in the other. The middle row mesh represents the upper part of a fire hydrant; it is not a revolution mesh and it evidently presents highly non-uniform section polylines in both grid directions. Finally, the vase mesh is obtained by scaled versions of one section polyline, shifted along the vertical axis with long and short shift steps. The considered meshes are characterized by highly non-uniform initial section polylines, so that the uniform scheme fails on all of them.

We have also tested NULISS on a wide set of data, acquired through a needle scanning device. We have reconstructed the data by means of NULISS and non-uniform tensor product bicubic spline surfaces. As shown in Figure 13, NULISS provides a faithful reconstruction, preserving the details present in the data. Oppositely, in some areas see, e.g., along the top border of the wings or top of head - tensor product splines generate more undulations than the ones present in the acquired data.

Finally, an application of NULISS was presented in [9]. In that work, NULISS was used to generate a surface starting from some of its feature curves, acquired through an interactive pen-like device (Fast Interactive Reverse Engineering). While the user interactively scans the feature curves, the surface underneath needs to change accordingly, so it is fundamental to have at disposal a quick method that at the same time allows for updating the surface shape and interpolating all the acquired data with satisfactory quality. In this context NULISS proved to be optimal in terms of 

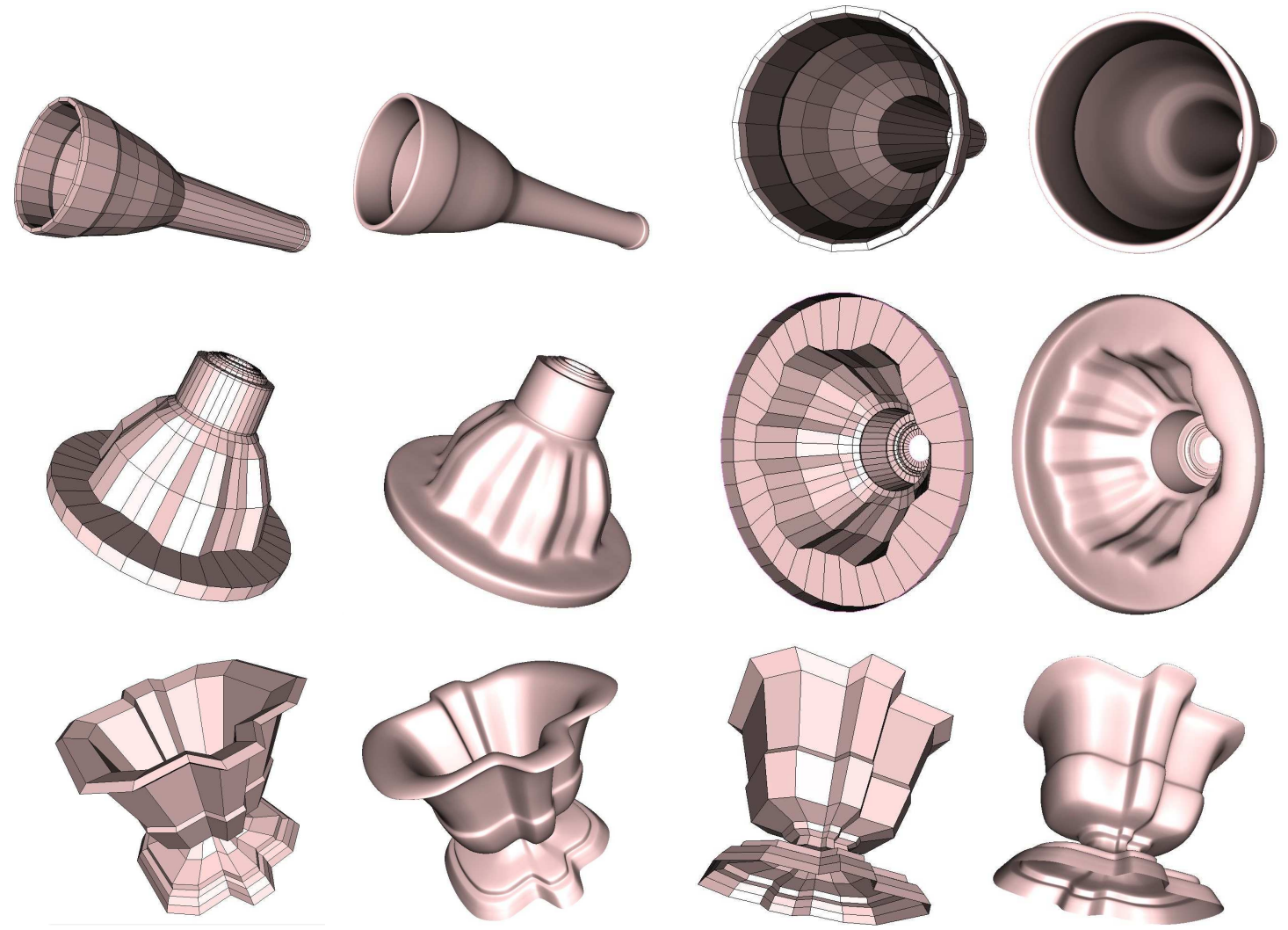

Figure 12: NULISS surfaces and the related initial meshes.

surface quality and speed of reconstruction, but none of its analytic properties were known, which was an additional motivation for the present paper.

\section{Summary and future work}

Non-uniform tensor product interpolants often give rise to significant undulation artifacts, due to the strict structure of the underlying parameterization. For this reason, the centripetal parameterization, that is proven to be optimal in the univariate case, does not significantly improve the behavior of the surface in the tensor product bivariate setting. In this paper, we have presented a novel class of non-uniform, non-tensor product, local interpolatory subdivision surfaces that generalizes non-uniform interpolatory 4-point schemes to regular quadrilateral meshes. This new proposal generates $C^{1}$-continuous limit surfaces with a better behavior than the well-established tensor product subdivision and spline representations. To a large extent, the advantage of this new construction is that a local parameterization is used, instead of a global average of the parameters, in such a way that each section curve is interpolated together with its parameters, thus maintaining its own centripetal parameterization. This allowed us to transpose to the surface setting the demonstrated benefits that the centripetal parameterization shows in curve interpolation.

Our future objective is the generalization of the proposed edge and face point subdivision rules to meshes with extraordinary vertices, pursuing the idea that the advantages of a suitable non-uniform parameterization, with respect to a uniform, might be significant also when interpolating meshes of arbitrary topology. 


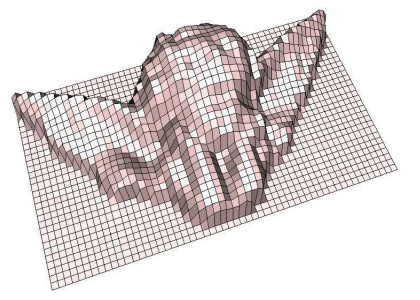

Initial data $(32 \times 57$ vertices $)$

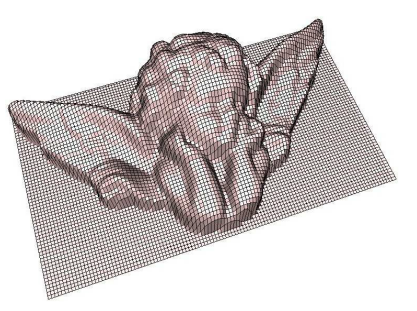

Initial data $(65 \times 114$ vertices $)$

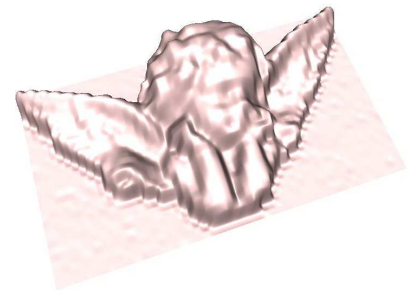

NULISS

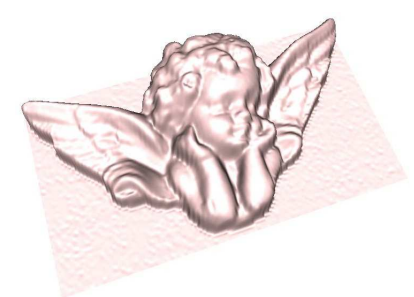

NULISS

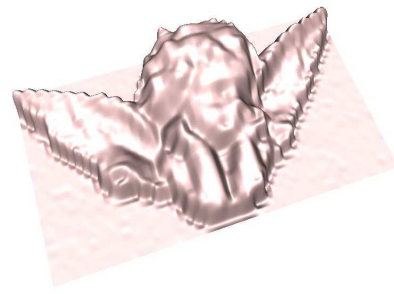

Tensor product

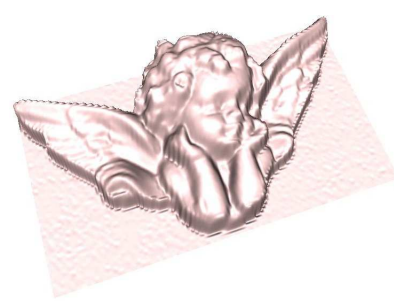

Tensor product

Figure 13: Reconstruction of scanned data through NULISS and non-uniform bicubic tensor product splines.

\section{Acknowledgements}

The authors would like to give special thanks to Nira Dyn, whose valuable hints and fruitful discussions enabled the completion of this work. The authors also thank Michael Floater for useful discussions and suggestions.

\section{References}

[1] Augsdörfer, U.H., Dodgson, N.A., Sabin, M.A., 2010. Variations on the four-point subdivision scheme. Comput. Aided Geom. Design 27(1),78-95

[2] Beccari, C., Casciola, G., Romani, L., 2007. A non-stationary uniform tension controlled interpolating 4-point scheme reproducing conics. Comput. Aided Geom. Design 24(1), 1-9.

[3] Beccari, C., Casciola, G., Romani, L., 2007. An interpolating 4-point $\mathrm{C}^{2}$ ternary non-stationary subdivision scheme with tension control. Comput. Aided Geom. Design 24(4), 210-219.

[4] Beccari, C., Casciola, G., Romani, L., 2009. Shape controlled interpolatory ternary subdivision. Applied Mathematics and Computation 215(3), 916-927.

[5] Beccari, C.V., Casciola, G., Romani, L., 2010. A unified framework for interpolating and approximating univariate subdivision. Applied Mathematics and Computation 216(4), 1169-1180.

[6] Beccari, C.V., Casciola, G., Romani, L., 2011. Non-uniform interpolatory curve subdivision with edge parameters built-upon compactly supported fundamental splines. BIT Numerical Mathematics 51(4), 781-808.

[7] Beccari, C.V., Casciola, G., Romani, L., 2011. Polynomial-based non-uniform interpolatory subdivision with features control. Journal of Computational and Applied Mathematics 235(16), 4754-4769.

[8] Beccari, C.V., Casciola, G., Romani, L., 2012. Construction and characterization of non-uniform local interpolating polynomial splines. Journal of Computational and Applied Mathematics, in press, doi:10.1016/j.cam.2012.06.025.

[9] Beccari, C.V., Farella, E., Liverani, A., Morigi, S., Rucci, M., 2010. A fast interactive reverse engineering system. Computer-Aided Design 42(10), 860-873.

[10] Cashman, T.J., Dodgson, N.A., Sabin, M.A., 2009. A symmetric, non-uniform, refine and smooth subdivision algorithm for general degree B-splines. Comput. Aided Geom. Design 26(1), 94-104.

[11] Conti, C., Gemignani, L., Romani, L., 2009. From symmetric subdivision masks of Hurwitz type to interpolatory subdivision masks. Linear Algebra and its Applications 431(10), 1971-1987.

[12] Conti, C., Gemignani, L., Romani, L., 2011. From approximating to interpolatory non-stationary subdivision schemes with the same generation properties. Adv. Comput. Math. 35(2), 217-241.

[13] Conti, C., Romani, L., 2010. Affine combination of B-spline subdivision masks and its non-stationary counterparts. BIT Numerical Mathematics 50(2), 269-299.

[14] Conti, C., Romani, L., 2011. Algebraic conditions on non-stationary subdivision symbols for exponential polynomial reproduction. Journal of Computational and Applied Mathematics 236(4), 543-556.

[15] Dahmen, W., Goodman, T.N.T., Micchelli, C.A., 1988. Compactly supported fundamental functions for spline interpolation. Numer. Math. $52,639-664$.

[16] Daubechies, I., Guskov, I., Sweldens, W., 1999. Regularity of irregular subdivision. Constr. Approx. 15(3), $381-426$. 
[17] Dyn, N., Floater, M., Hormann, K., 2009. Four-point curve subdivision based on iterated chordal and centripetal parameterizations. Comput. Aided Geom. Design 26(3), 279-286.

[18] Dyn, N., Levin, D., 1995. Analysis of asymptotically equivalent binary subdivision schemes. J. Math. Anal. Appl. 193, 594-621.

[19] Handbook of Computer Aided Geometric Design, Gerald Farin, Josef Hoschek, Myung-Soo Kim, Eds. Elsevier, 2002.

[20] Floater, M., 2008. On the deviation of a parametric cubic spline interpolant from its data polygon. Comput. Aided Geom. Design 25(3), 148-156.

[21] Floater, M., Beccari, C., Cashman, T., Romani, L., 2012. A smoothness criterion for monotonicity-preserving subdivision. Adv. Comput. Math., in press, doi:10.1007/s10444-012-9275-y.

[22] Müller, K., Reusche, L., Fellner, D., 2006. Extended subdivision surfaces: Building a bridge between NURBS and Catmull-Clark surfaces. ACM Transactions on Graphics 25(2), 268-292.

[23] Romani, L., 2009. From approximating subdivision schemes for exponential splines to high-performance interpolating algorithms. Journal of Computational and Applied Mathematics 224(1), 383-396.

[24] Romani, L., 2010. A circle-preserving $C^{2}$ Hermite interpolatory subdivision scheme with tension control. Comput. Aided Geom. Design 27(1), 36-47.

[25] Schaefer, S., Goldman, R., 2009. Non-uniform subdivision for B-splines of arbitrary degree. Comput. Aided Geom. Design 26(1), 75-81.

[26] Sederberg, T.W., Zheng, J., Sewell, D., Sabin, M., 1998. Non-uniform recursive subdivision surfaces. Proceedings of the 25th annual conference on Computer Graphics and Interactive Techniques, pp. 387-394.

[27] Warren, J., 1995. Binary subdivision schemes for functions over irregular knot sequences. In: M. Dæhlen, T. Lyche, L.L. Schumaker (Eds.), Mathematical Methods in CAGD III, pp. 543-562, Vanderbilt University Press, Nashville (TN)

[28] Yuksel, C., Schaefer, S., Keyser, J., 2009. On the parameterization of Catmull-Rom curves. Proceedings of ACM Joint Conference on Geometric and Physical Modeling, pp. 47-53. 